\title{
Full-Scale CFD Investigations of Helical Strakes as a Means of Reducing the Vortex Induced Forces on a Semi-Submersible
}

\author{
Vegard Holland, Tahsin Tezdogan*, Elif Oguz \\ Department of Naval Architecture, Ocean and Marine Engineering, Henry Dyer Building, University \\ of Strathclyde, 100 Montrose Street, Glasgow, G4 0LZ, UK \\ ${ }^{*}$ Corresponding author; e-mail: tahsin.tezdogan@strath.ac.uk, phone: +44(0)1415484532
}

\begin{abstract}
As the search for oil in the Gulf of Mexico (GoM) moves into deeper waters, floating platforms such as semi-submersibles are in increasing demand. As semi-submersibles increase in size, the effect of vortex-induced motions (VIMs) becomes a significant problem in their design. VIMs stem from transverse forces caused by the current affecting the platform, with vortexes moving downstream on either side of the structure. The loop/eddy current phenomenon in the GoM leads to a constant current being present in the area, with speeds of up to $1.8 \mathrm{~m} / \mathrm{s}$. The accurate prediction of the vertical and transverse motions of semi-submersibles is crucial for the design of the riser systems. It is therefore beneficial to investigate the hydrodynamic forces acting on the geometry, and means of reducing these forces. A common method of reducing transverse forces is the addition of column appendages, such as helical strakes. In this paper, full-scale computational fluid dynamic analyses are carried out to examine the transverse forces caused by this vortex shedding using realistic current velocities in the GoM. Helical strakes are attached to the geometry to break up the coherence of the vortex shedding and the performance of these strakes is investigated numerically.
\end{abstract}

Keywords: Vortex-Induced Motion, Semi-submersible, CFD, Helical strake

\section{Introduction}

When oil drilling moved into the offshore waters in the early 1900s, floating drilling rigs which were able to move around the waters were required. Until the 1950s, monohull ships were used to conduct these drilling activities, but they were found to have significant motions in waves. Following this, the industry sought more stable drilling platforms for offshore drilling, and in the 1960s the first semisubmersibles were introduced.

A semi-submersible offers a large flexible work platform for various offshore activities like drilling rigs, production platforms and accommodation units. These activities benefit from small motion responses as they all have strict safety regulations and motion restrictions potentially adversely 
affecting uptime. Drilling rig downtime is often caused by a combination of excessive vessel motions in the transverse direction as well as heave motions in the vertical direction. For production semisubmersibles, the heave and transverse motions (sway) are the key parameters for the design of the riser and mooring systems, driving both cost and complexity of the riser and mooring system. It is therefore critical to investigate the hydrodynamic forces affecting the structures and to investigate means for reducing such forces.

The Gulf of Mexico (GoM) is an ocean basin located between the United States in the north and west, Mexico in the south and Cuba in the east. The GoM sits on huge deposits of oil and gas, and is one of the largest petroleum producing areas in the United States. Most natural resources discovered are located in deep waters, requiring floating drilling and production platforms. The GoM experiences a phenomenon of so-called loop/eddy currents, which occurs when Caribbean water enters from the south, does a "loop" within the Gulf, and exits in the east where it joins up with the Gulf Stream. This leads to constant currents being present in the GoM, with speeds reaching up to $1.8 \mathrm{~m} / \mathrm{s}$, affecting all platforms located within the gulf.

All structures submerged in a fluid flow are affected by vortex induced motions/vibrations depending on the Reynolds number $(R e)$. These motions are the result of oscillating surface pressures induced by vortex shedding on either side of the structure. The motion in a transverse direction to the flow is dominant compared to the inline direction. The vessel responses caused by vortex-induced motions (VIMs) can therefore be narrowed down to two dominant directions; surge and sway. The magnitude of the forces created by the vortexes are very dependent on the diameter of the structure. In contrast to the conventional semi-submersibles having slender columns, 'deep draft semi-submersibles' have large diameter columns, and are therefore more affected by the VIM phenomenon compared to the conventional ones. As mentioned by Kim et al. (2015) the prediction of VIM is a difficult task due to the complex behaviour of vortex structure shedding and their interactions with a structure in higher Reynolds numbers.

Through the years many different methods have been used to determine the motions of a semisubmersible floating in a geographical area. Sengupta and Chatterjee (1986) proposed a simple analytical method to predict the motion responses of a semi-submersible to regular waves. They reported that their numerical results were in good agreement with the experimental results. Another way of determining vessel motions is model testing, as carried out in Lundgren and Berg (1982). They performed experimental studies with a four-column semi-submersible drilling rig in regular and irregular seas measuring motions and resistance in currents and waves. The model scale was 1:65 and the model was moored with catenary mooring lines. Their study explicitly showed the effect of the metacentric height of the rig on the motion responses of the platform. As clearly explained in Rijken and Leverette (2008), the crucial parameters determining the VIM responses in tank tests are the 
reduced velocity, Froude and Reynolds numbers. Of the three parameters, the reduced velocity is the most critical one which is used to assess the experiments. Rijken and Leverette (2008) highlight the biggest issue associated with model experiments is the scaling such that the lower Reynolds number of the model compared to the prototype may undergo a different viscous flow regime which could lead to a different response between the model and prototype. They also state that vortex shedding location is independent of heading and almost independent of current speed. Their study showed that there is no significant change in vortex generation in model tests due to location of appurtenances. This is due to fact that the Reynolds number is not expected to alter the location of vortex generation in the model. Conversely, as explained in Rijken and Leverette (2008) Reynolds number is still crucial in determining the other viscous effects i.e. drag load. In order to avoid any scaling effects, a full-scale model is used for all simulations reported in this paper. Around the same time, Roddier et al. (2009) experimentally investigated the effect of Reynolds number and hull appurtenances (such as chains, pipes and anodes) on VIMs using a vertically moored truss spar model with strakes. They performed three sets of experiments at varying headings and reduced velocities to address the scale issue inherited in any experiments.

Recently a number of experimental studies have been carried out to determine the vortex-induced motions of a semi-submersible. To give an example from the published literature, Hong et al. (2008) conducted seakeeping model test experiments with a deep-draft semi-submersible (DDS) to predict its motion characteristics in varying wave, wind and current conditions. Their findings revealed that a DDS may undergo significant VIM in the direction normal to the current. Their experimental results also showed that the amplitude of the VIM is significantly affected by the current speed and the wave excited particle velocity. Later, Gonçalves et al. (2012) presented an experimental study outlining the effects of current heading and hull appendages on the VIM of a semi-submersible by measuring its surge, sway and yaw amplitudes. They presented their findings in detail in their paper with an aim to show which maximum motions are experienced in each mode of motion.

Armin and Srinil (2013) presented a mathematical fluid-structure interaction modelling and analysis of two flexibly-mounted circular cylinders arranged in tandem and subject to fluid cross flows in their paper. They used two different semi-empirical wake oscillator models based on the van der Pol and Rayleigh equations to determine the hydrodynamic lift and buffeting forces and their time variations. They predicted the response amplitude behaviours using a direct numerical time integration approach and they also carried out a parametric study to determine the vortex- and wake-induced vibration transverse response of the two interfering upstream and downstream cylinders. Their study led to the development of another thorough study on the vortex-induced vibration (VIV) modelling of cylinders as reported in Armin (2016), who carried out a series of experimental and numerical studies to assess the effect of spacing between two cylinders on the VIV response of each cylinder. Armin used two experimental configurations using identical and non-identical cylinders at various speeds which 
indicates the effect of $R e$ on the cylinders' response. Armin also predicted the effect of natural frequency on the behaviour of non-identical cylinders using two sets of similar structures. Finally, Armin made some suggestions for future research in this area. We believe that in this paper we are addressing one of Armin's future recommendations by carrying out a detailed computational fluid dynamics (CFD) work focusing on the effect of strakes on the flow characteristics and VIM responses of a multi-column cylinder.

As an alternative to experimental methods, CFD-based methods have been used to accelerate research devoted to semi-submersibles. Recent developments in computer technology have enabled CFD methods to be utilised to thoroughly analyse VIM problems pertaining to semi-submersibles. The research in this area includes the interactions of the vortexes caused by one column which affects the flow characteristics of the columns behind it. These are very complex simulations, though fortunately it is possible to run such simulations in a reasonable amount of time with today's computational resources. Tan et al. (2014a) attribute the main reason behind the popularity of CFD methods to the fact that CFD methods are capable of simulating specific conditions such as full-scale Reynolds number effects which cannot be determined experimentally.

Tan et al. (2014b) numerically investigated the effect of hull appurtenances on the VIM of a 'tension leg platform' (TLP) designed for the Southeast Asian region. In order to check the accuracy of the CFD calculations, experiments were also performed using a 1:70 scale model of their TLP which is composed of circular columns. Their results showed that the measured sway responses have a small peak for $5<U r<8$ in the case of a hull with appurtenances at $22.5^{\circ}$ current heading where VIM usually occur. This indicates that VIM behaviour of a hull depends on current heading. Both CFD calculations and experiments showed that the response amplitudes are lower in the case of appurtenances than the hull without appurtenances for the same reduced velocities. Their results showed that the VIM responses are not significant for their TLP model. It is worth noting that the results obtained in their study are constrained within the specific TLP design which has a shallow draft and short wetted column length above the spokes so the results cannot be generalised.

It is critical to be able to predict the effect of appurtenances on the VIM performance of a floating offshore structure using the real current environment which it will experience. Tan et al. (2014a) carried out numerous CFD simulations to determine the effect of appurtenances on the VIM performance of a TLP which was designed for Borneo in South China. They aimed to investigate the effects of reduced velocity, current heading and appurtenances on the TLP VIM performance. The correlation with CFD calculations and experimental results was presented and the effectiveness of the appurtenances was discussed. Their results demonstrated that the mean drag coefficients of the hull with and without appurtenances are very close and different current headings give different VIM motions. 
Kim et al. (2015) performed experimental and numerical studies using a paired-column semisubmersible model for different current speeds and headings. They reported that the biggest challenge in CFD simulation of VIMs is the validation of the employed numerical methods due to the scarcity of the experimental data. They also highlighted that the Reynolds number in the model-scale is two orders of magnitude less than the Reynolds number in the full-scale. As their study reports a detailed validation and verification study of a typical VIM simulation, their paper is heavily consulted for this study's time-step and mesh generation resolutions as explained in Section 3.

Ma et al. (2013) reported that the actual severity of VIM in a real-life measurement is much less than predicted using full-scale CFD analysis. This proves that the results presented in this paper should be considered as a worst-case scenario where no restoring forces are present.

The attachment of strakes or other appendages to reduce VIM has been thoroughly studied for sparplatforms by many researchers such as Irani and Finn (2004, 2005), Roddier et al. (2009) and Wang et al. (2009). For example, Irani and Finn (2005) carried out a wide range of tests at a 1/40 model scale in order to investigate the effectiveness of different strake designs to reduce the Truss Spar VIV response. Two configurations were carried out to investigate the impact of strake pitch on VIV motions. Experiments were performed at the Force Technology's tow tank laboratory in Lyngby, Denmark in 2005. The tank dimensions are reported as $240 \mathrm{~m} \mathrm{x} 12 \mathrm{~m}$ x $5.4 \mathrm{~m}$. These studies mentioned above explicitly state that helical strakes attached to the curves of the geometry can be very efficient in reducing VIM if designed correctly.

Lefevre et al. (2013) presented extensive VIM CFD calculations using Star-CCM+ for a spar system in their paper. They compared their CFD predictions to those obtained from the experimental results. They performed turbulence model, mesh and time step sensitivity studies and showed the best resolutions for their CFD simulation. It is finally reported that their study can be taken as guidelines for VIM CFD calculations for a spar system.

As newer semi-submersibles are getting larger in size, they are more exposed to VIM. It is therefore becoming more necessary to investigate the magnitude of the transverse forces acting on them and to investigate the methods to decrease the magnitude of excited forces. This study therefore aims to numerically assess the performance of helical strakes attached to the columns of a semisubmersible as a means of reducing the forces acting in a normal direction to the current. A Detached Eddy Simulation (DES)-based CFD method was used to perform the numerical simulations reported in this paper. In order to avoid scale effects, the analyses were carried out in the full-scale. As seen in the literature review, most of the numerical studies in this field were carried out with a model-scale geometry, hence our numerical results obtained for a full-scale semi-submersible model may be of interest to academics and practitioners working in this field when designing such appendages aiming to reduce VIMs and VIVs. 
The semi-submersible geometry was initially modelled using the Dassault Systemes' 3D-modelling software SolidWorks using their educational licence. This program allowed us an accurate generation of the semi-submersible geometry. The geometry was then imported into Star-CCM+, version 11 . Star-CCM+ is a CFD-based finite volume code developed by CD-Adapco (2016). Following this, the CFD simulations were run for various combinations of geometry and current speeds utilising the high performance computer facilities at the University of Strathclyde, Glasgow. Details of the simulations completed in this work are given in the end of Sub-Section 2.1.

This paper has been organised as follows. Section 2 gives background theory to VIM and vortex shedding problems. Afterwards, the numerical setup of the CFD model is explained, with details provided in the contained sub-sections in Section 3. Following this, in Section 4, all of the results obtained from this work, including a verification study, are presented and discussed. Finally, the main results drawn from this study are briefly summarised, and suggestions are made for future research in Section 5.

\section{Background theory}

\subsection{Gulf of Mexico loop/eddy currents}

As warm Caribbean water enters the Gulf of Mexico from the south, it performs a loop within the Gulf before exiting just south of Florida to join up with the Gulf Stream. This loop varies in size and strength, but may reach up to Louisiana, located at the northernmost part of the Gulf. The loop current will occasionally shed a rotating ring of hot water called "loop eddy currents" (or "LC Eddy") as seen in Figure 1. 


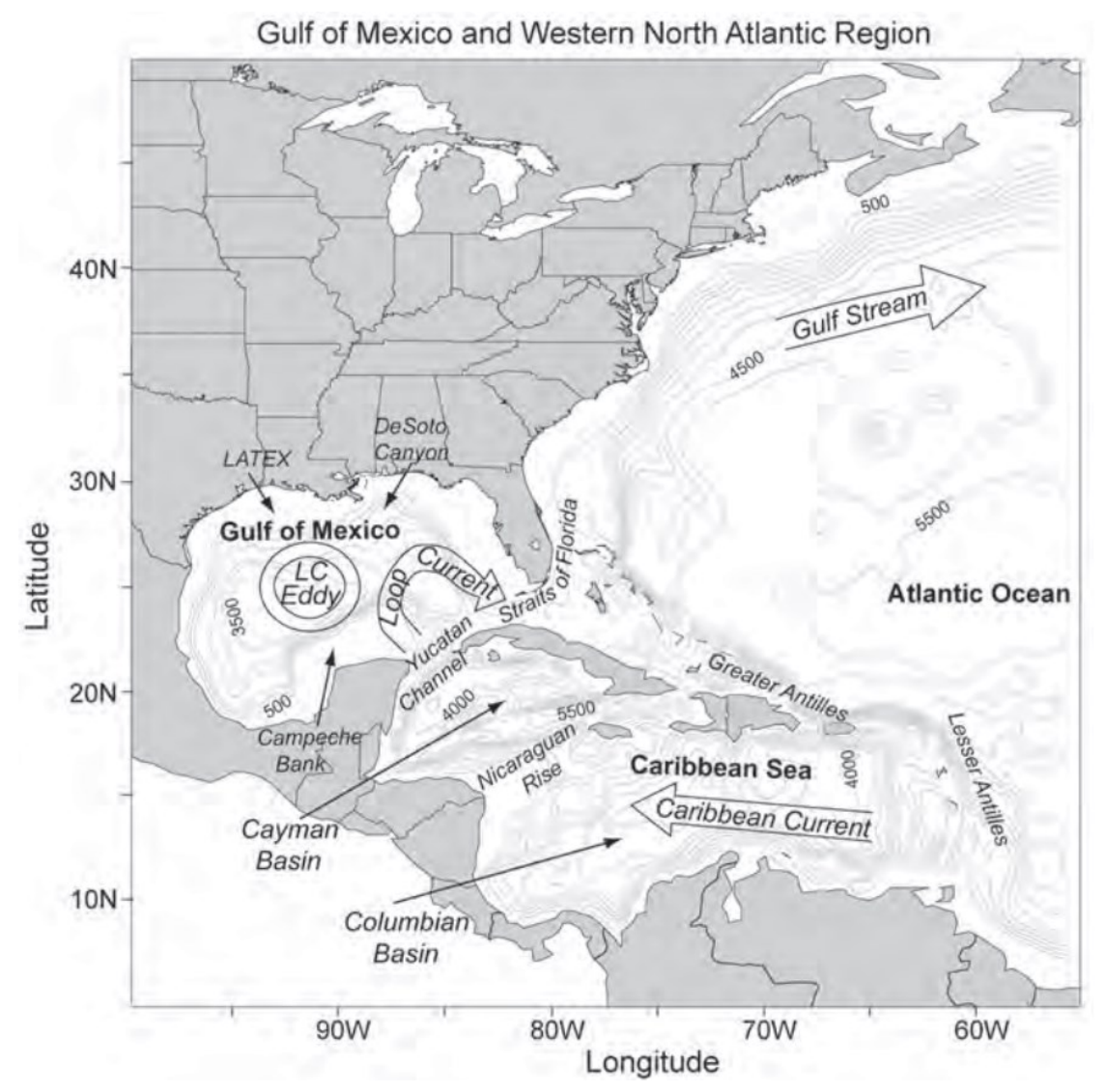

Figure 1 Map of the Gulf Mexico and Caribbean waters showing the Loop Currents, Loop Eddy Currents and Gulf Stream (Oey et al., 2002)

The loop current is the dominant feature of the water circulation in the Gulf of Mexico with peak speeds of 1.5-1.8 m/s. The loop/eddy currents are vital in the fuelling and generation of hurricanes that frequently wreak havoc in the area. As this is an area of deep waters and rich deposits of oil and gas, there are many floating production and drilling platforms like semi-submersibles in the area, all affected by the loop/eddy currents.

The currents that affect the semi-submersible will mostly be loop/eddy currents. In the numerical calculations, three velocities $(0.5 \mathrm{~m} / \mathrm{s}, 1.0 \mathrm{~m} / \mathrm{s}$ and $1.5 \mathrm{~m} / \mathrm{s})$ were selected to model the most prominent and consistent velocities in the area. It is necessary to note that all the current conditions were applied for a zero degree of heading angle.

\subsection{Flow regime}

To understand the flow behind a structure submerged in water affected by a current it is important to have an understanding of the non-dimensional Reynolds number:

$$
R e=\frac{V D}{v}
$$

where $V$ is the flow velocity, $D$ the structure/cylinder diameter (or characteristic length) and $v$ the kinematic viscosity of the fluid. When the Reynolds number increases, the flow characteristics around 
and behind the structure change, going through different stages depending on the type of forces acting on the structure. The different regimes behind a tube depending on the fluid $R e$ are shown in Figure 2.

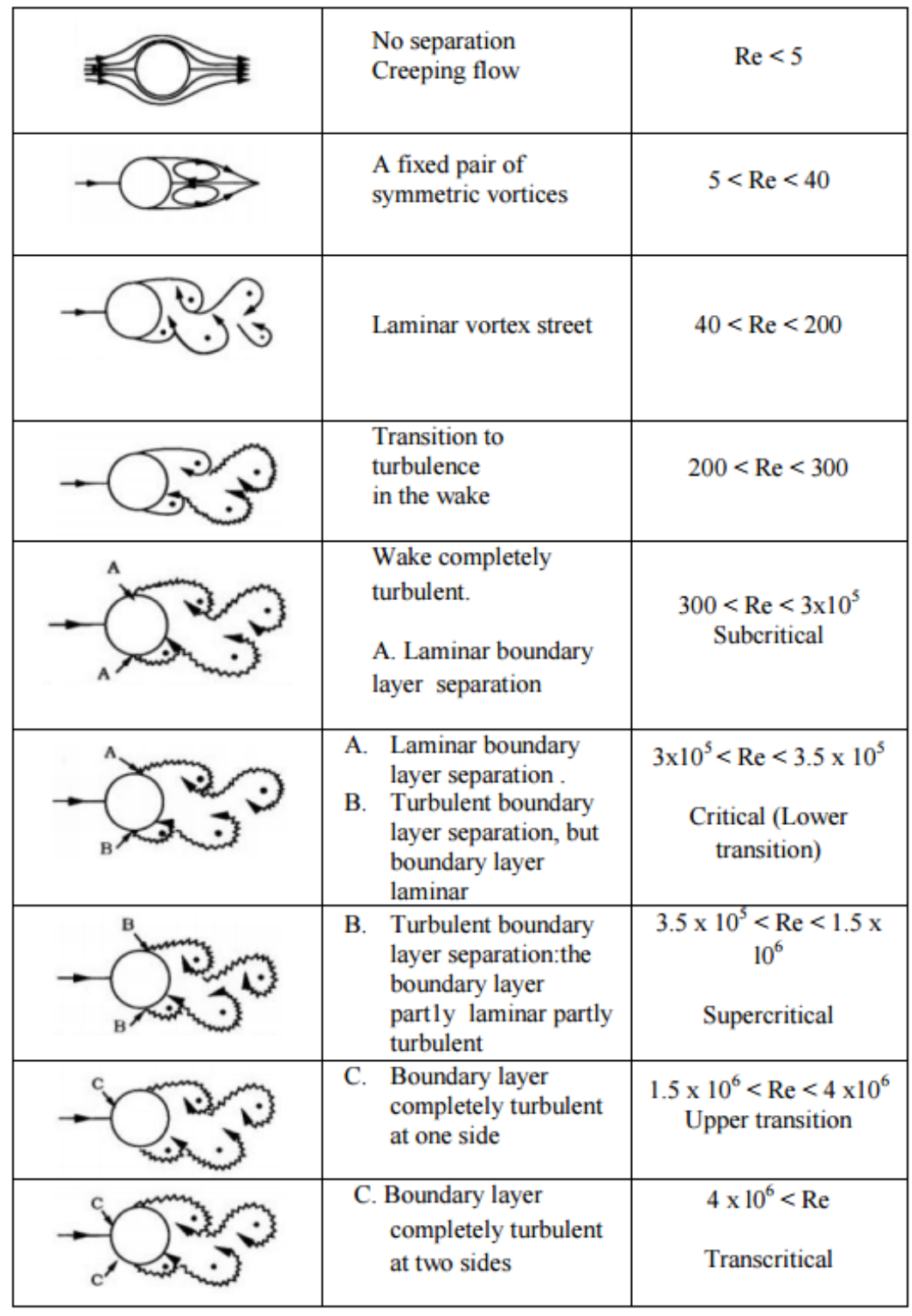

Figure 2 Regimes of fluid flow behind a smooth tube (Sumer and Fredsøe, 1997)

As shown in Figure 2, separation of the flow from the cylinder begins at $R e=5$. In the region $5<R e<40$ two fixed vortices will appear in the immediate flow of the cylinder. When increasing the flow speed to the region of $40<\operatorname{Re}<150$, transverse forces start affecting the cylinder. This is caused by the phenomenon of vortex shedding where vortices are shed alternately from each side of the cylinder, generating cyclical transverse forces acting to the cylinder. When increasing Re the vortex street changes from laminar to turbulent and in the region $300<\operatorname{Re}<3 \times 10^{5}$ the vortex street is fully turbulent. In this region the flow regime is called the subcritical flow regime.

In the flow regime of $3 \times 10^{5}<\operatorname{Re}<3.5 \times 10^{6}$ the boundary layer becomes turbulent at the separation point. This happens at only one side of the cylinder but it does alternate non-cyclically. This leads to one side 
being turbulent at the separation point, and the other laminar. This is called the critical flow regime and results in a narrower and disorganized wake. The turbulent vortex street is re-established in the region $R e>3.5 \times 10^{6}$. This vortex street is very similar to the ones found for lower Reynolds number, but with a turbulent flow "disturbing" it. This is the region where most large structures (large diameters) that are placed in open water are found. This last regime is called the transcritical flow regime.

\subsection{Vortex shedding}

The most important and relevant effect mentioned in the previous section is the vortex-shedding phenomenon. The vortex shedding is caused by the incoming flow "rolling into" a shear layer (Figure 3) that has been formed downstream of the cylinder. This shear layer is created by the separation of the boundary layer over the geometry surface due to the adverse pressure gradient. This pressure gradient is in turn created by the divergent flow at the back of the cylinder.

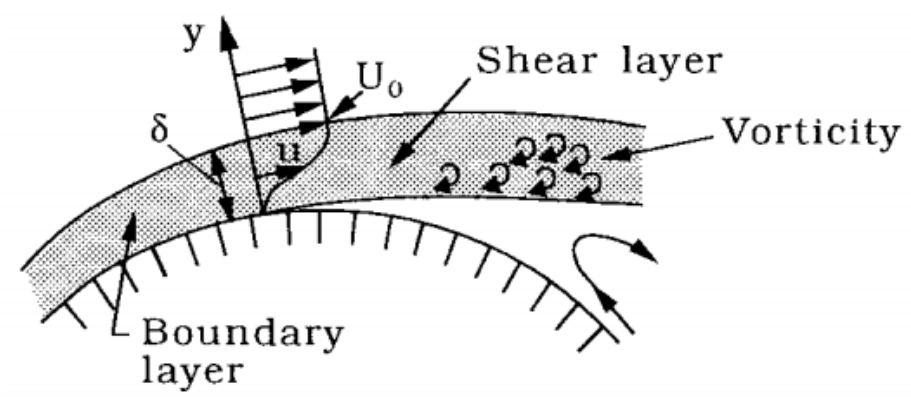

Figure 3 Detailed picture of flow near separation (Sumer and Fredsøe, 1997)

The point of separation is defined as where the shear stress reduces to zero. This is shown in Figure 4, where a positive pressure gradient $d p / d x>0$. This pressure gradient makes the velocity profile close to the geometry more and more S-shaped, eventually causing separation.

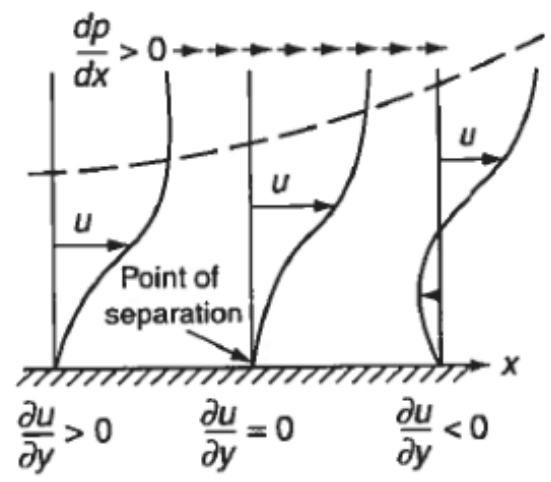

Figure 4 Point of separation (White, 2006)

The mechanism of vortex shedding is a continuous occurrence in the wake of the cylinder, alternately shedding vortexes from each side of the cylinder. It is described in Figure 5. 
a)

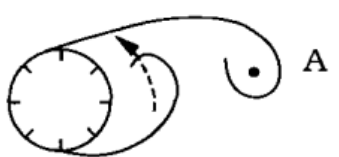

$\mathrm{B}$ b)

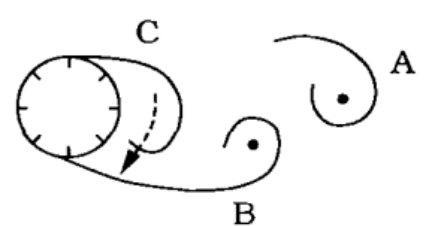

Figure 5 Mechanism of vortex shedding (Sumer and Fredsøe, 1997)

Sumer and Fredsoe (1997) explain the mechanism of vortex shedding as quoted below:

“... the larger vortex (Vortex A in Figure $5 a$ ) is likely to become strong enough to draw the opposing vortex (Vortex B) across the wake. The vorticity in Vortex $A$ is in the clockwise direction (Figure $5 b$ ), while that in Vortex $B$ is in the anti-clockwise direction. The approach of vorticity of the opposite sign will then cut off further supply of vorticity to Vortex A from its boundary layer. This is the instant where Vortex $A$ is shed. Being a free vortex, Vortex $A$ is then convected downstream by the flow. Following the shedding of Vortex $A$, a new vortex will occur at the same side of the cylinder, so-called Vortex $C$ (Figure 5b). Vortex $B$ will now play the same role as Vortex A, namely it will grow in size and strength so that it will draw Vortex $C$ across the wake (Figure $5 b$ ). This will lead to the shedding of Vortex B. This process will continue each time a new vortex is shed at one side of the cylinder where the shedding will continue to occur in an alternate manner between the sides of the cylinder."

This alternating motion will lead to transverse forces attempting to move the structure in the transverse direction. If the structure is allowed to move such as in the case of semi-submersibles or spar platforms, this can lead to damage to equipment like the riser systems, which have strict movement limitations.

\subsection{Boundary layer}

As explained in Section 2.3, vortex shedding is caused by the incoming current being affected by the boundary layer of the geometry. As the thickness of the boundary layer is very thin, the number of cells needed in a computer model in order to simulate this is very high. As an accurate measurement of the simulated boundary layer the dimensionless wall distance was developed and is defined as

$$
y^{+}=\frac{u^{*} d}{v}
$$

where $u^{*}$ is the friction velocity calculated from the wall shear stress $(\tau)$ at the nearest wall, $d$ is the wall distance and $v$ is the kinematic viscosity of the fluid. By definition, the wall shear stress magnitude is calculated by $\tau_{w}=\rho u^{* 2}$ in which $\rho$ is the fluid density.

\subsection{Drag and sway forces}

In response to the periodic change in the vortex shedding, the pressure distribution around the wetted geometry will change periodically. This will lead to periodic alterations in the force components acting on the structure. In this paper two different force components will be measured and presented for varying current speeds. These forces involve the force in the direction of the current flow (drag force) and the force in the normal direction of the current flow (sway force). The latter one acts in the 
transverse direction causing VIMs as discussed earlier. These forces are commonly represented by their non-dimensional forms, by the so-called force coefficients. A force coefficient is calculated by dividing the force magnitude to the product of $\left(0.5 \rho A U^{2}\right)$, where $A$ is the cross-sectional area of the structure.

\subsection{Helical strakes}

To reduce the motions caused by the vortex shedding that can damage equipment and induce a fatigue failure, modes of altering the geometry that the fluid passes over is one approach. It is possible to mitigate VIV by streamlining the structure. Passive control options for suppressing VIV are given in Figure 6 as taken from Blevins (1990) and Kwon et al. (2002).

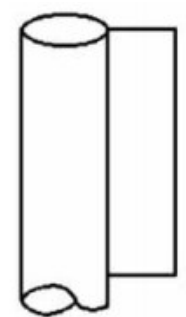

(a)

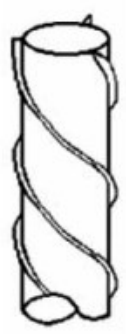

(e)

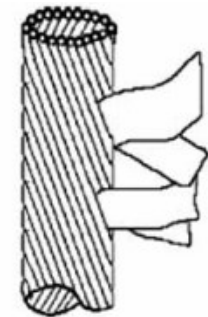

(b)

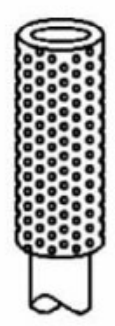

(f)

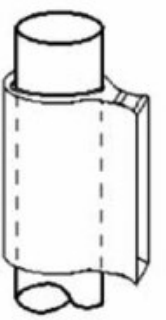

(c)

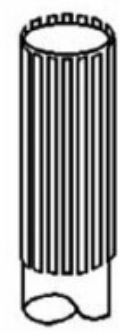

(g)

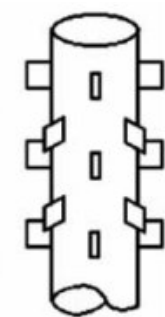

(d)

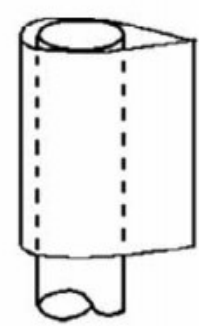

(h)

Figure 6 Passive control options for supressing (Blevins, 1990 and Kwon et al. 2002) 
One method of altering the geometry is introducing Helical Strakes that are welded on the curvatures of the geometry. Historically it has been used to reduce transverse forces on high chimney stacks and process towers, and when used offshore in the design of spar-platforms, and is shown to be extremely efficient, reducing the transverse movements by up to $80 \%$ (Kumar et al., 2008).
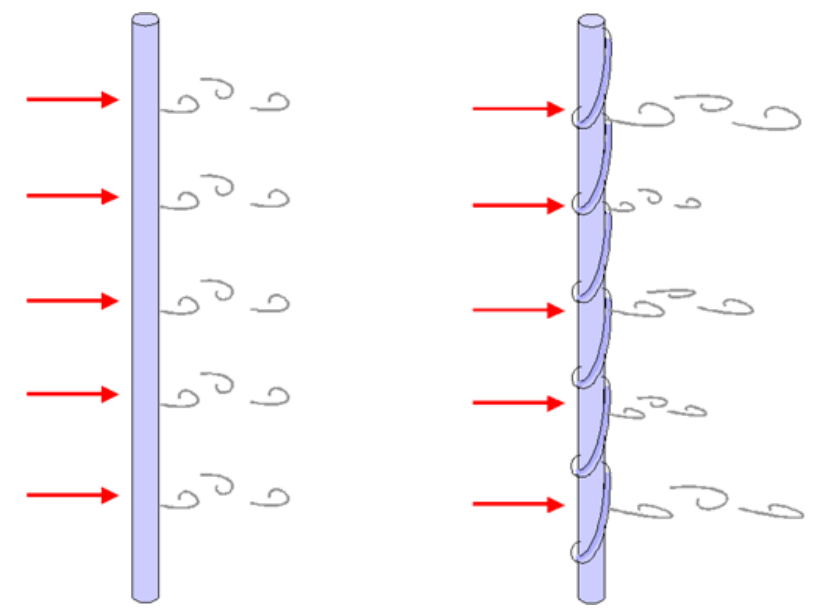

Figure 7 Vortex shedding without and with helical strakes (Dirac Delta Consultants Ltd, n.d.)

As shown in Figure 7, helical strakes work by breaking up the coherence of vortex shedding along the column, thereby reducing the cyclic transverse forces affecting the structure. However the change in geometry of the structure might increase the overall drag. Numerical studies mimicking experiments are also conducted using CFD in order to allow for correlation between the experimental and numerical results. As reported in Atluri et al. (2006), to assess the performance of helical strakes, CFD analyses were performed on spar-platforms with helical strakes appended. They carried out two series of tests for three different models. Hexahedral mesh was used in Model I and the geometry was selected similar to Halkyard et al. (2005)'s previous work. The second Model includes holes on the strakes and its appendage whereas Model III includes a truss section. The first set of tests aimed to determine the reduced velocity range from $4 \mathrm{~m} / \mathrm{s}$ to $10 \mathrm{~m} / \mathrm{s}$ for a heading of $150^{\circ}$. The second series of tests were performed using different current headings for a constant current velocity of $7 \mathrm{~m} / \mathrm{s}$ in order to determine the impact of current heading on response. Their numerical results revealed the effect of including the appurtenances for different cases. For more detailed results, reference may be made to Atluri et al. (2006)'s paper.

\section{Numerical set-up}

Up to this point, this paper has provided a background to the study and has given an introduction to the research reported in this paper. This section will present details of the numerical modelling and techniques used in this work. 


\subsection{Geometry}

The semi-submersible model was created in a piece of 3D CAD software with the dimensions presented in Table 1. It has four square columns with four pontoons connecting them, all with rounded edges. It is worth noting that only the underwater part of the geometry was modelled in this study.

Table 1 The dimensions of the semisubmersible in the full-scale, given in metres

\begin{tabular}{|l|l|l|}
\hline Part & Item & Value (m) \\
\hline \multirow{4}{*}{ Semi-submersible } & Draft & 21.00 \\
\cline { 2 - 3 } & Column C/C longitudinal & 67.50 \\
\cline { 2 - 3 } & Column C/C transverse & 67.50 \\
\hline \multirow{4}{*}{ Columns } & Column length & 17.80 \\
\cline { 2 - 3 } & Column width & 17.80 \\
\cline { 2 - 3 } & Column bilge radius & 3.00 \\
\cline { 2 - 3 } & Column transition height & 6.60 \\
\hline \multirow{5}{*}{ Pontoons } & Pontoon transition length & 3.325 \\
\cline { 2 - 3 } & Pontoon width & 17.80 \\
\cline { 2 - 3 } & Pontoon bilge radius bottom & 1.125 \\
\cline { 2 - 3 } & Pontoon bilge radius top & 1.125 \\
\cline { 2 - 3 } & Pontoon height & 8.75 \\
\hline
\end{tabular}

A three-dimensional model of the semi-submersible geometry used in this study is shown in Figure 8 . The geometry illustrated in the figure is a common semi-submersible model used in the Gulf of Mexico.

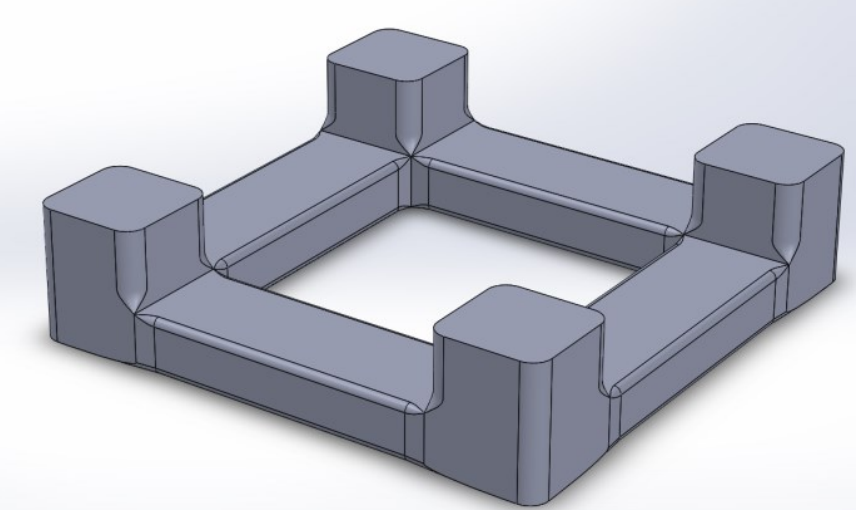

Figure 8 Three-dimensional model of the semi-submersible

The helical strakes attached to the columns for the second batch of simulations use dimensions taken from spar-platforms that operate in the same area, under the same loop/eddy currents. These strakes have been shown to be very effective, reducing the transverse forces of the spar-platforms by up to $80 \%$, as mentioned in Section 2.6. The dimensions used in the semi-submersible model were taken from Atluri et al. (2006). The width of the strakes was calculated to be $13 \%$ of the column width $(0.13 \times 17.80=2.3 \mathrm{~m})$, as proposed by Atluri et al. (2006). Figure 9 shows one of the four identical columns with a helical strake covering each curve. 
It is worth noting that in this study the helical strakes were placed at the rounded column corners as this is the site where significant vortex shedding occurs independent of the angle of attack of the current. The vortexes are generated as they follow the curvature of the rounded corner and are shed. The idea is therefore to disrupt this shredding using helical strakes and allow for the flow to separate differently through the height of the column.

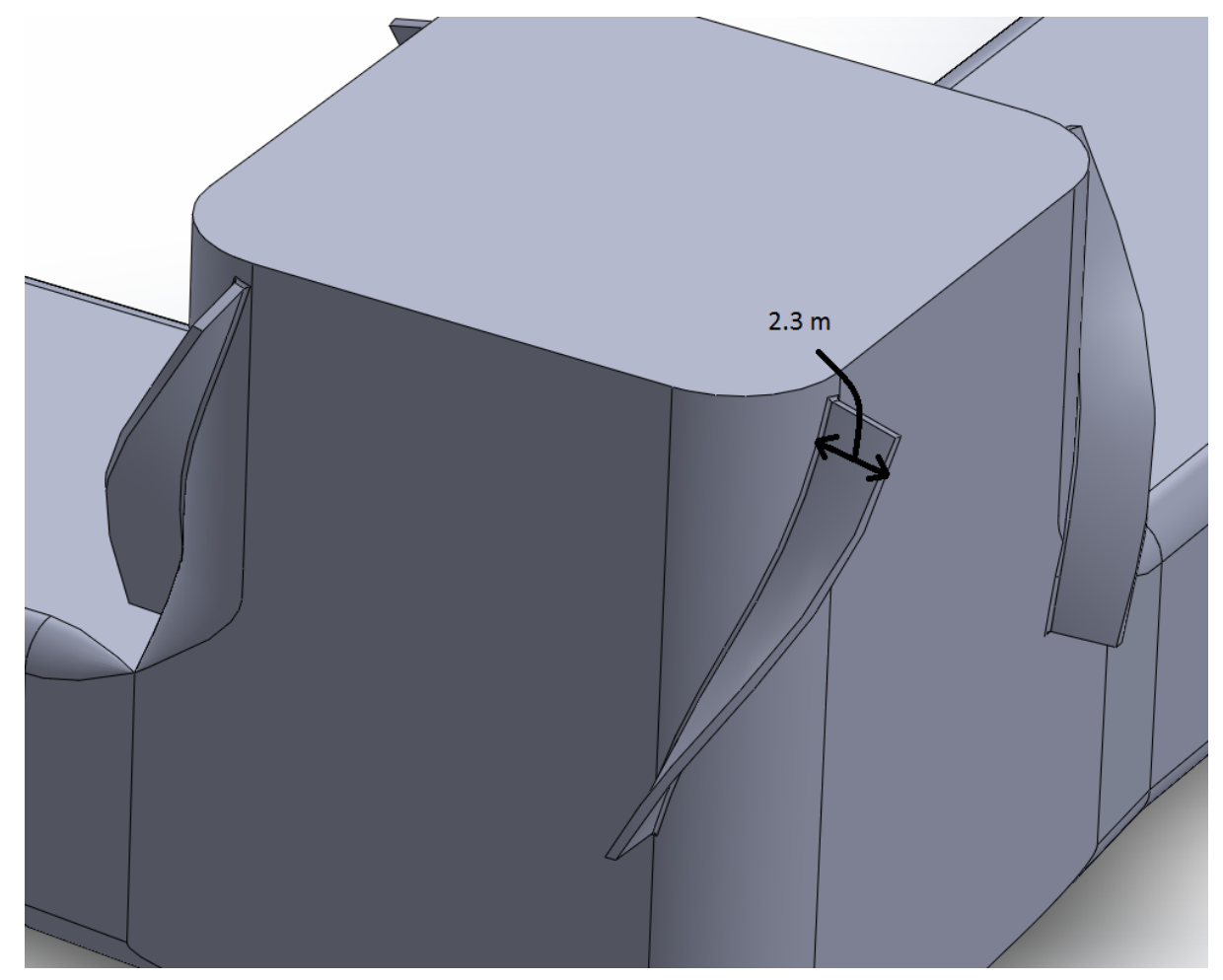

Figure 9 One of the four columns with helical strakes showing the width of the helical strakes

\subsection{Physics modelling}

The physical models define variables like fluid and flow data, turbulence models and the numerical methods adopted in this study to solve the governing equations. To realistically simulate vortex shedding on a full-scale geometry in a reasonable simulation time it is of great importance to select the correct physical models.

Due to the high demand of computational power that a typical Large Eddy Simulation (LES) requires, a hybrid model called Detached Eddy Simulation (DES) has been developed and initially presented in Spalart et al. (1997). This model treats near-wall regions with the Reynolds Averaged Navier-Stokes (RANS) model while using the LES for the rest of the flow. This model was originally developed by replacing the distance function $d$ in the Spalart-Allmaras (SA) function with a modified distance function $\tilde{d}=\min \left[d, C_{D E S} \Delta\right]$ where $C_{D E S}$ is a constant while $\Delta$ is the largest dimension of the grid cell being solved. This simple modification altered the model completely, causing the model to behave as a RANS model in regions of small cells (close to walls) and in a Smagorinsky (LES) manner for the rest of the model. Similar to a previous CFD-based study published by Tan et al. (2014b), in this study the 
DES approach was applied in all CFD simulations. An illustration of the theory behind the DES method is given in Figure 10. As also stated in Tan et al. (2014b) this method employs the all $\mathrm{y}^{+}$wall treatment on the boundary layers. The all $\mathrm{y}^{+}$wall treatment is a hybrid model, which provides a more realistic approach than the low-Re or the high-Re treatments. To calculate shear stress, this wall treatment uses blended wall laws, which present a buffer region that suitably blends the laminar and turbulent regions together. The result is similar to the low-Re $\mathrm{y}^{+}$treatment as $\mathrm{y}^{+} \rightarrow 0$ and similar to the high-Re $\mathrm{y}^{+}$treatment for $\mathrm{y}^{+}$values greater than 30 (Tezdogan, 2015 and CD-Adapco, 2016). Since a full-scale model was used in this study, the $\mathrm{y}^{+}$values on the geometry were relatively high.

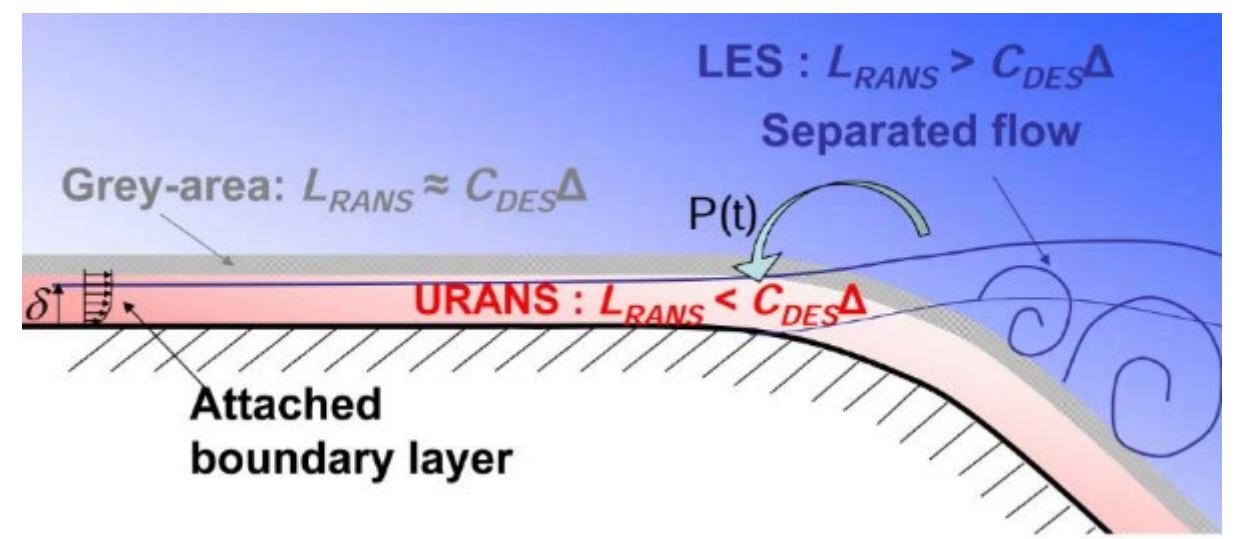

Figure 10 Sketch of RANS and LES regions in a DES approach (Sagaut et al., 2013)

In addition to the above, in this study's CFD work the Implicit Unsteady model is used with the segregated flow to control the update at each physical time for the calculation along with the time-step size. In this model, each physical time-step is controlled by some number of inner iterations to achieve the convergence of the solution in that given time-step. The number of inner iterations set in this study was 10 which was deemed to be sufficient to reduce the residuals by three to four orders of magnitude at each time-step. The same resolution was also adopted by Tan et al. (2014b). It should also be noted that the second-order temporal scheme was adopted to solve the transit term of the governing equations. In addition, a liquid model was activated in the CFD software to represent a single pure liquid substance.

\subsection{Computational domains and boundary conditions}

The walls of the computational domain must be given physical properties to define the boundary conditions of the simulation. Figure 11 delineates that a velocity inlet boundary condition was set in the negative $\mathrm{x}$-direction to model the current fluid flow in the computational domain. In the downstream direction (the positive $\mathrm{x}$-direction) a pressure outlet boundary condition was applied to fix the static pressure at the outlet. As can be seen from Figure 11, the other boundaries were set as a symmetry plane. It should be recapped that the first derivative of the velocity and shear stress are zero at the symmetry plane. The boundary condition on the semi-submersible model was designed as a noslip wall to ensure that the relative fluid velocity tangential to the wall was zero which is an important 
factor in order to achieve any vortex shedding caused by shear stress in the boundary region. It should also be noted that when a no-slip wall is set, normal stress on the wall remains zero, as mentioned in Fergizer and Peric (2002).

Taking precedence from similar previous studies (Kim et al., 2011, Tan et al., (2013, 2014a, 2014b) and $\mathrm{Xu}$ et al., 2012), the locations of the boundaries relative to the semi-submersible's centre of gravity $(\mathrm{Co} G)$ were determined based on the overall length of the structure $(C)$ as illustrated in Figure 12.

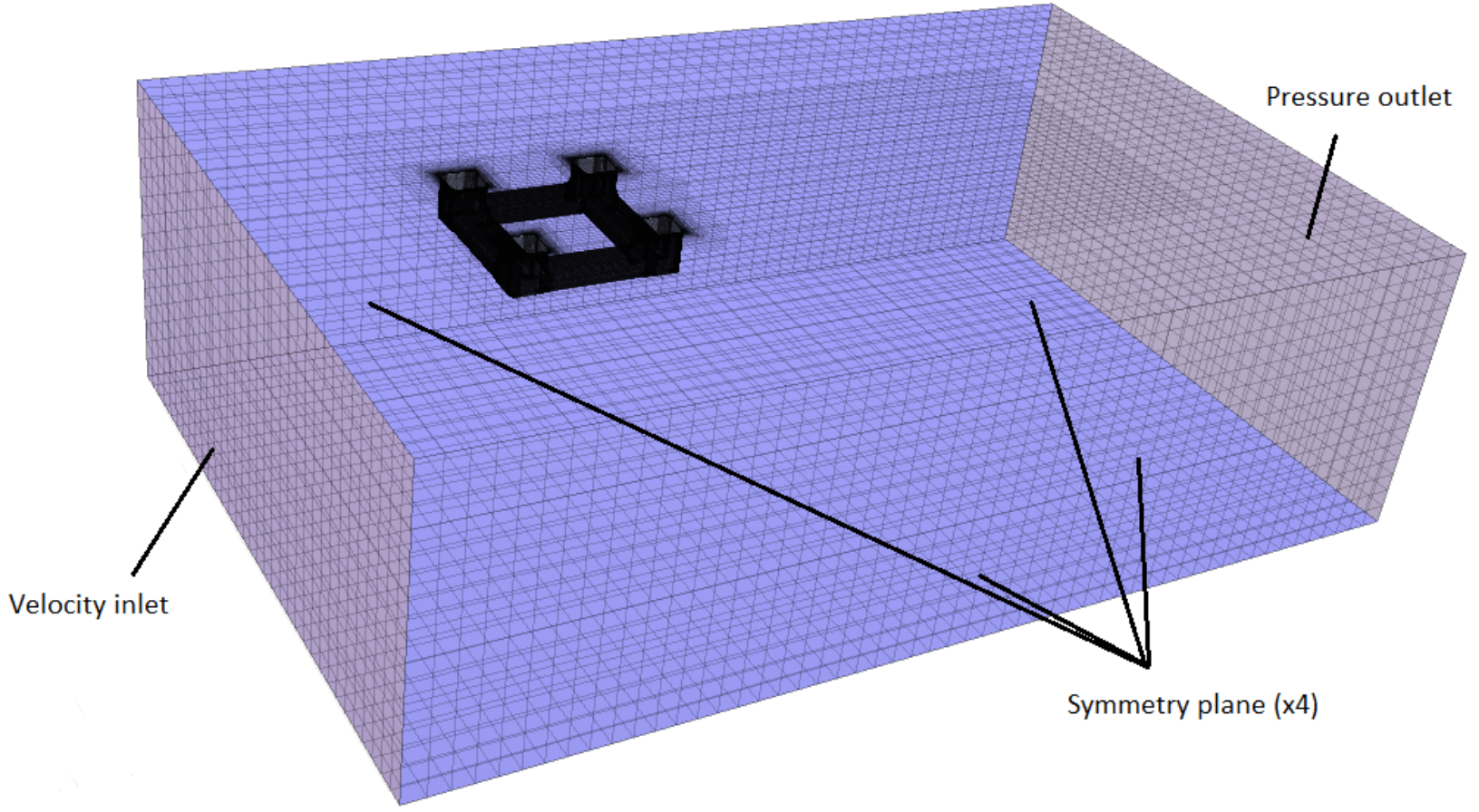

Figure 11 Notations of boundary conditions applied to the walls of the computational domain 


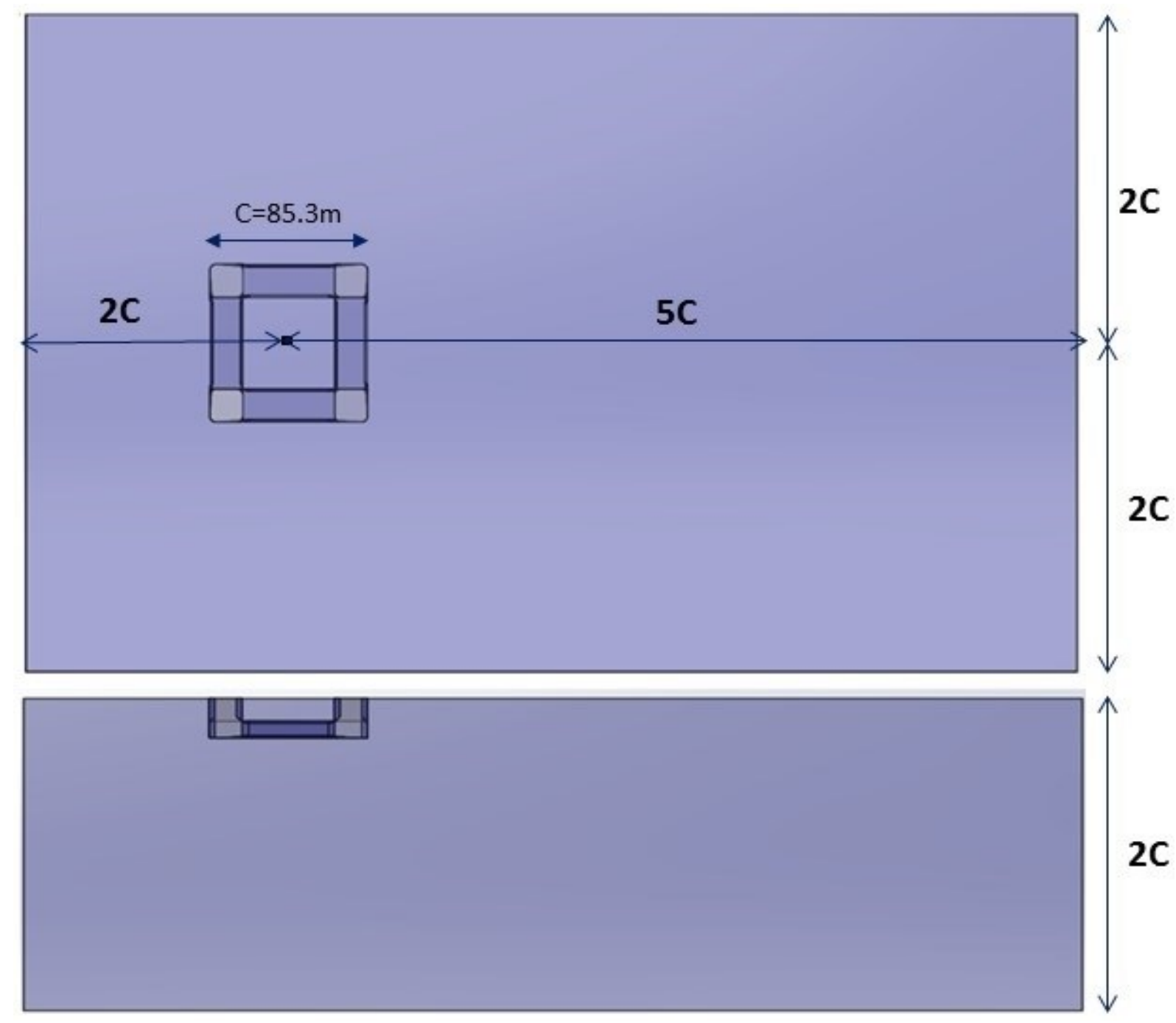

Figure 12 The dimensions of the computational domain using top (the above figure) and side views (C: overall length of the model)

\subsection{Time-step selection}

The Courant number (CFL), which is the ratio of the physical time step $(\Delta t)$ to the mesh convection time scale, relates the mesh cell dimension $\Delta x$ to the mesh flow speed $U$ as given below:

$$
C F L=\frac{U \Delta t}{\Delta x}
$$

The Courant number is typically calculated for each cell and should be less than or equal to 1 for numerical stability.

Often, in implicit unsteady simulations, the time step is determined by the flow properties, rather than the Courant number. In order to gain a suitable level of accuracy within a reasonable running time, the time step size is determined by $\Delta t=0.0042 L / U$ (where $L$ is the length of equal sides of the column) as proposed by Nishino et al. (2008), who performed numerical studies of fluid around a circular cylinder. 


\subsection{Mesh generation}

As mentioned in Section 1, the geometry is imported from SolidWorks into Star-CCM+. The mesh was generated using Star-CCM+'s automatic meshing facility, which resulted in an approximate total of 6.4 and 6.8 million cells for a simulation without and with strakes appended, respectively.

\subsubsection{Mesh generation without helical strakes}

To capture the complex flow around the semi-submersible, a number of volumetric controls were used in Star-CCM+ to refine the mesh in such specific areas. A cross-sectional view inside the computational domain showing the refined mesh areas can be seen in Figure 13. The mesh consists of hexahedral cells with various sizes with a prismatic layer close to the surface to capture the boundary layer effect causing the vortex shedding.

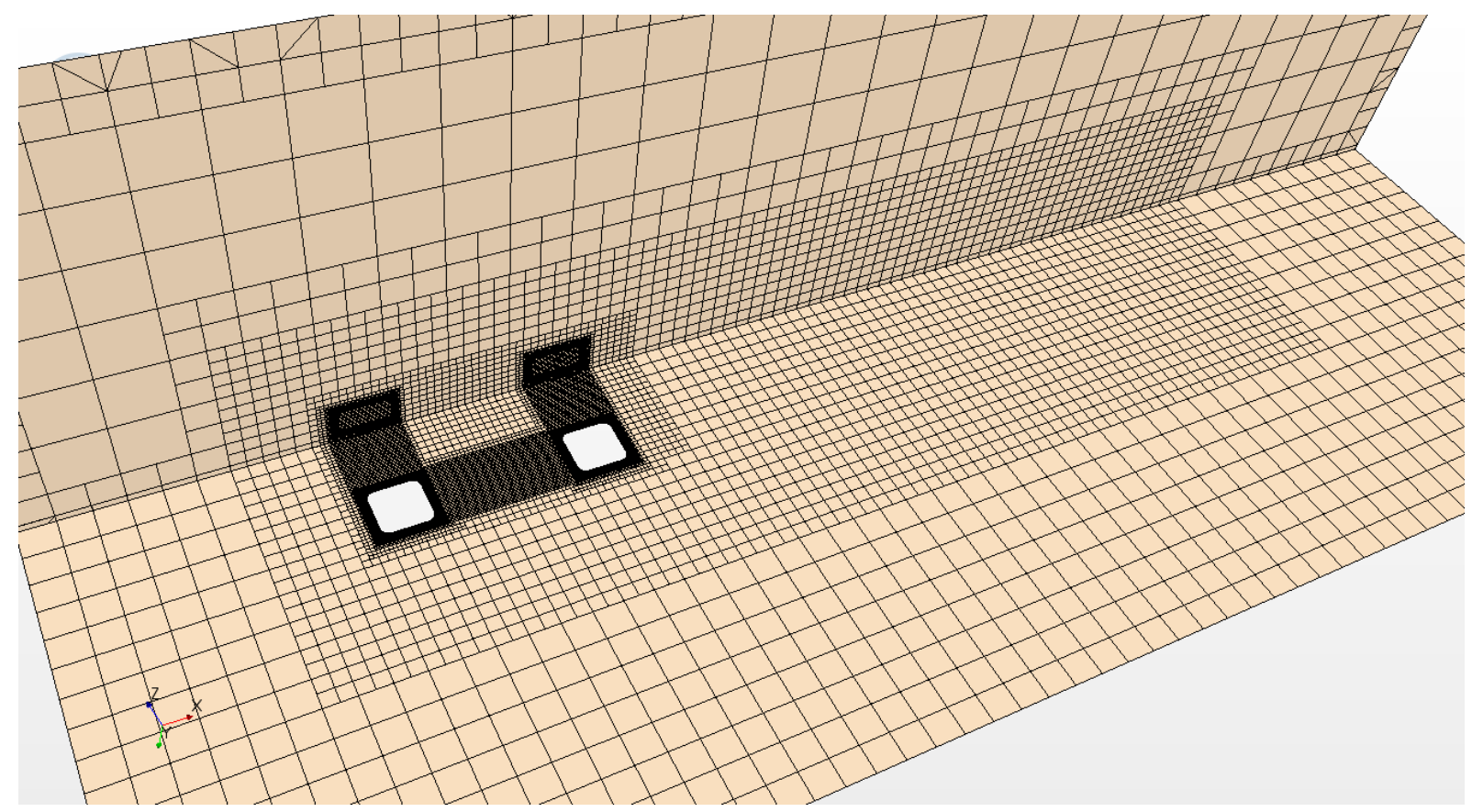

Figure 13 A cross-sectional view of the mesh generated inside the computational domain 


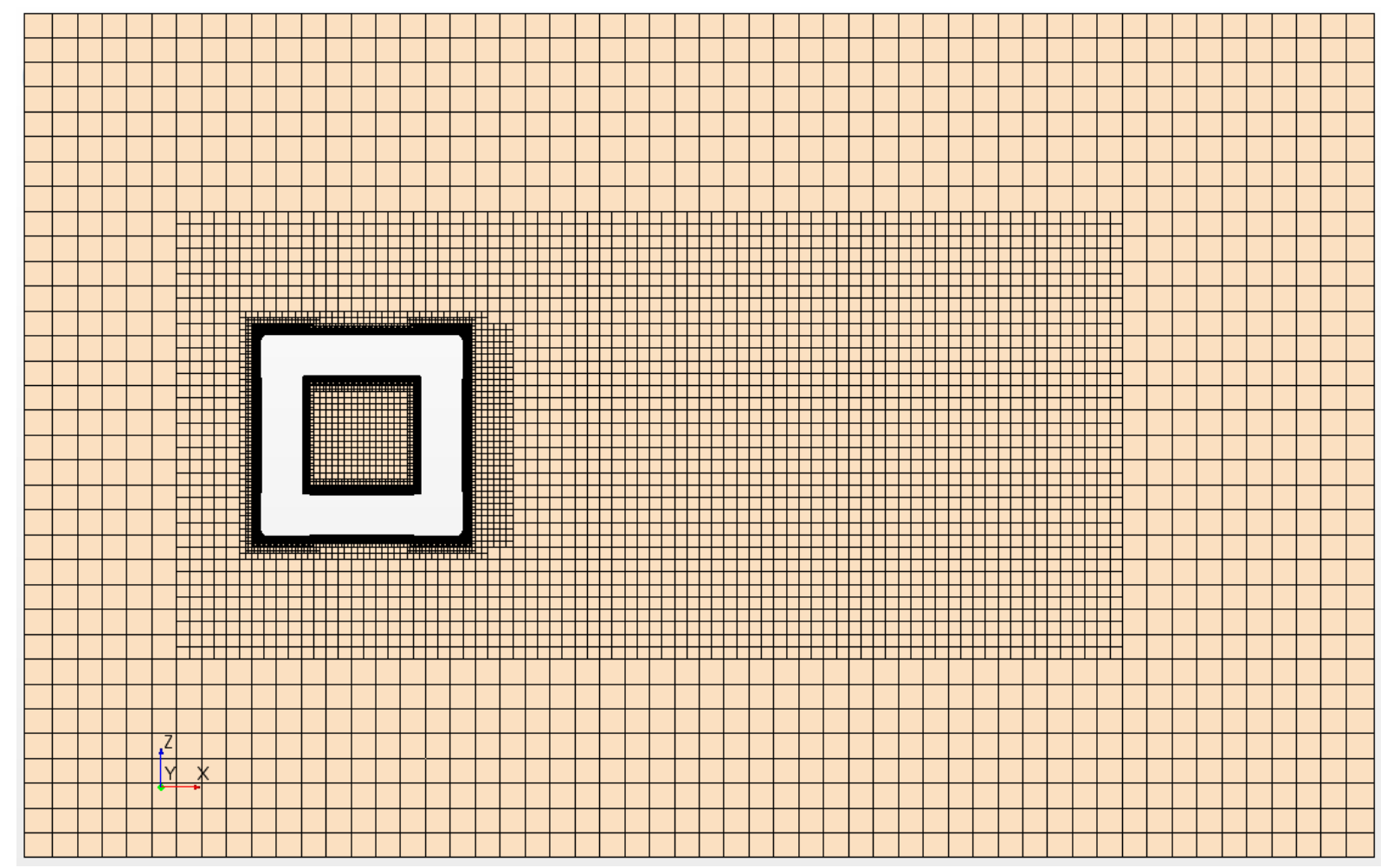

Figure 14 A cross-section of the computational mesh showing refined areas around the geometry

As Figure 14 shows, refined meshes were used to capture various aspects of the flow. To capture the flow in the wake of the semi-submersible, a refined mesh was also applied. A finer mesh was constructed around the geometry to capture the flow between the columns and in the immediate wake of the second columns. This is where the most prominent vortexes are expected to occur. To achieve a smaller courant number around the geometry, a refined mesh was constructed around each column and pontoon, as well as an even more refined mesh around each column corner. This increased the stability and convergence of the model when the simulations were running. Figure 15 shows the threedimensional computational mesh-grid with one of the columns in focus. The blue mesh illustrates the mesh on the water surface while the grey cells illustrate the mesh on the geometry surface. 


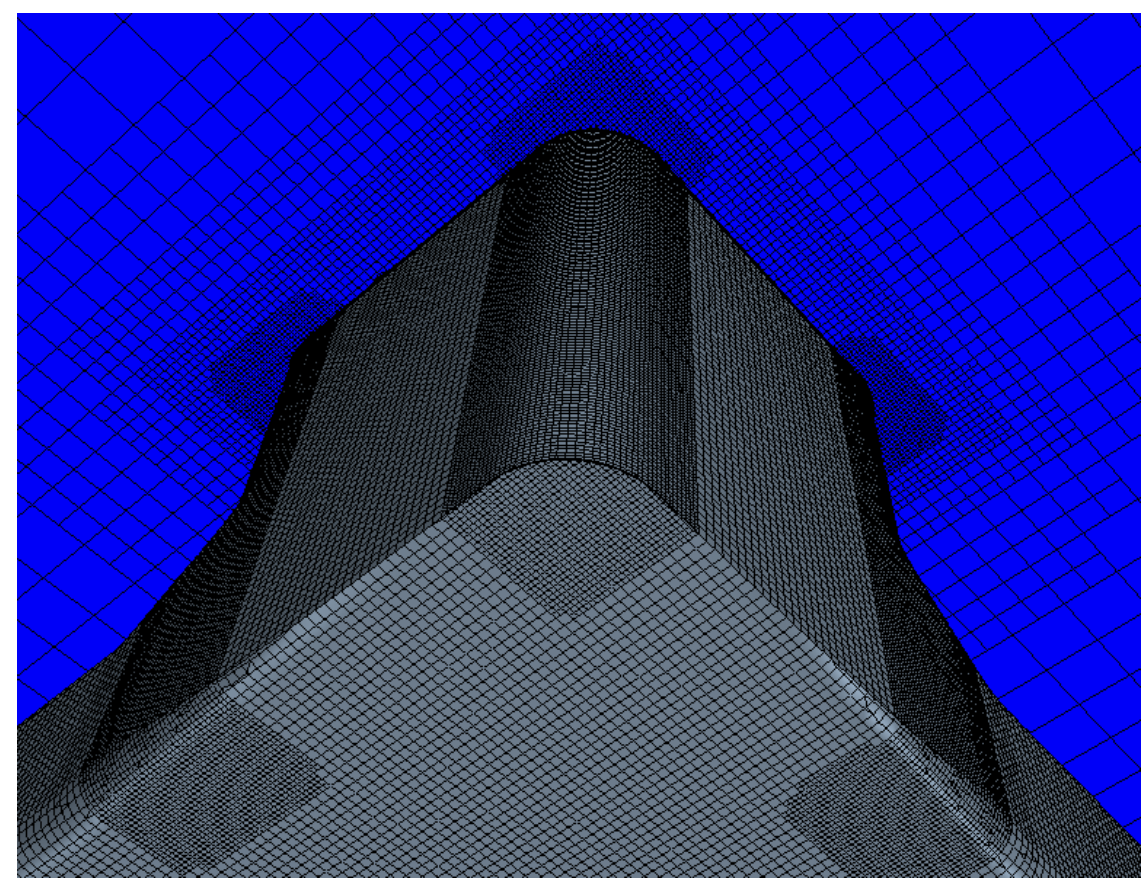

Figure 15 A view of the computational mesh without helical strake

To recreate the boundary layer around the geometry a prism layer was applied. The prism layer had a total thickness of $0.1 \mathrm{~m}$ and consisted of 24 layers within this thickness. This ensured that the shear forces causing the vortex shedding were captured and that the cell size closest to the geometry was sufficiently thin to achieve a $\mathrm{y}^{+}$value of around 1 . The prism layer representing the boundary layer is shown in Figure 16. 


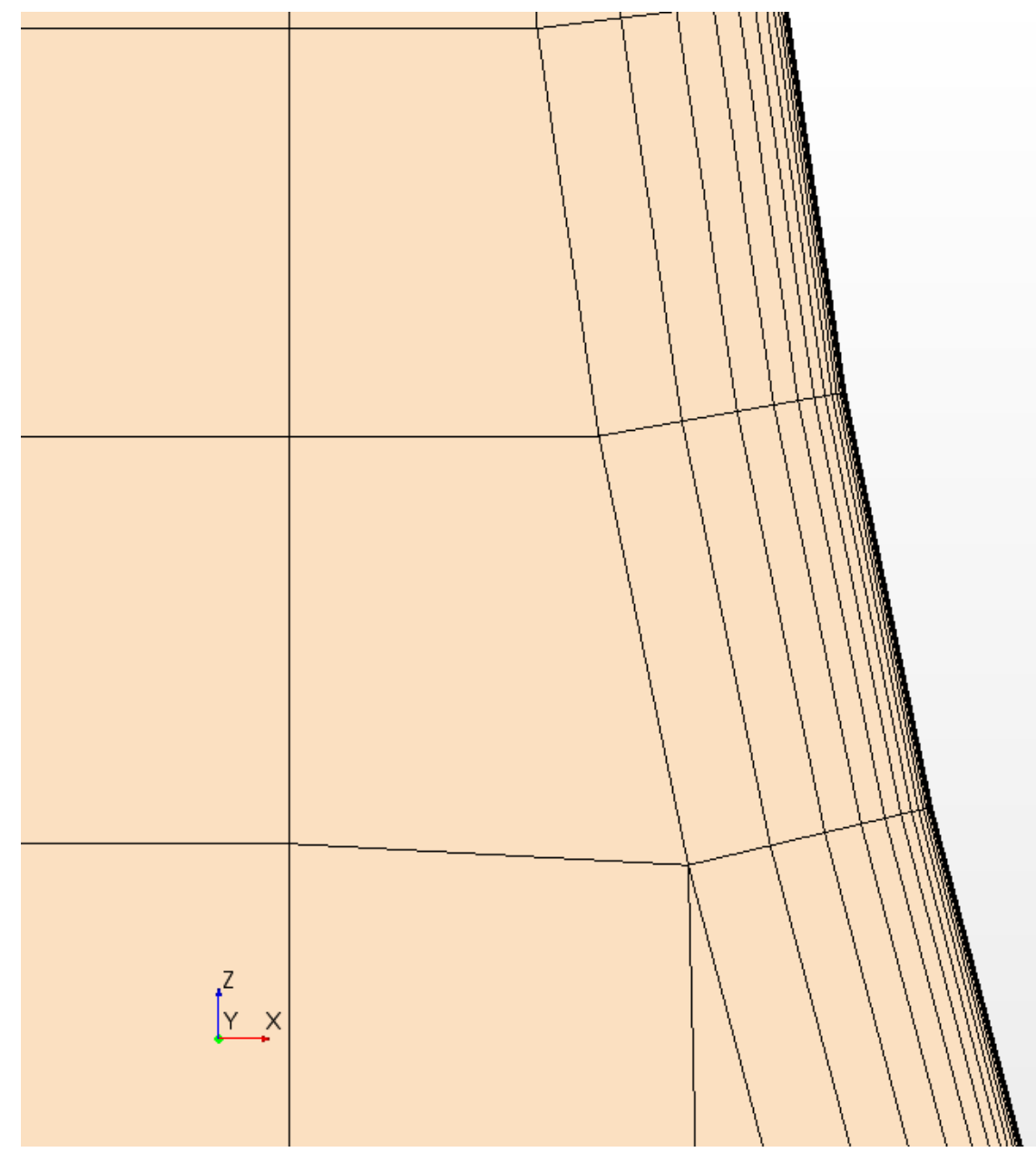

Figure 16 Prism layer close to geometry curves (cross-section at=13 m)

\subsubsection{Mesh generation for the geometry with helical strakes}

The surface and volumetric mesh was generated in the same fashion as explained in Section 3.5.1. The only difference in terms of meshing is the increased wetted surface due to the extra geometry. The three-dimensional computational mesh grid is shown in Figure 17. As mentioned earlier, the blue mesh represents the mesh at the water level while the grey cells represent the mesh on the geometry. The prism layer generation around the strakes is shown in Figure 18. 


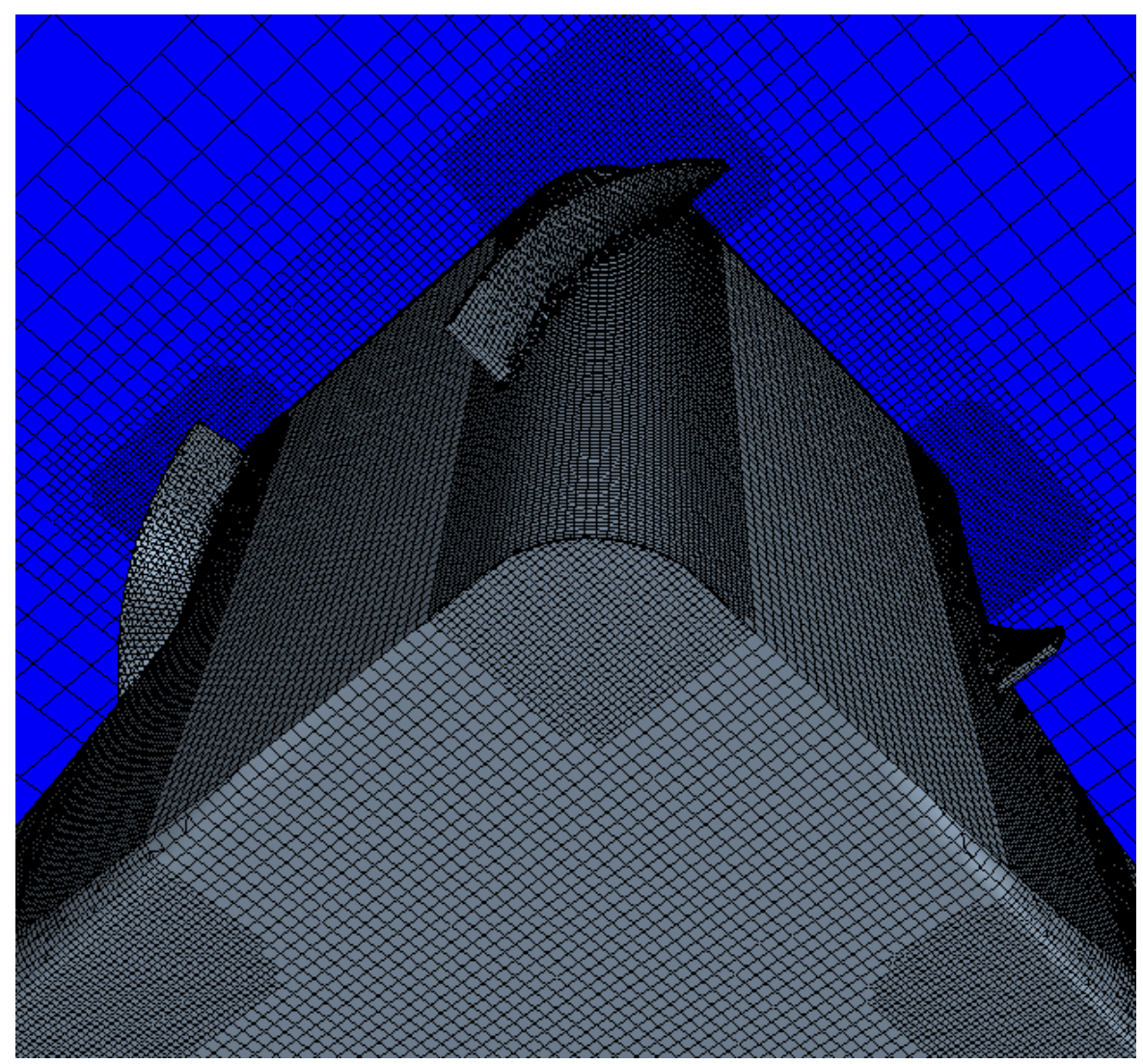

Figure 17 A view of the computational mesh with helical strake

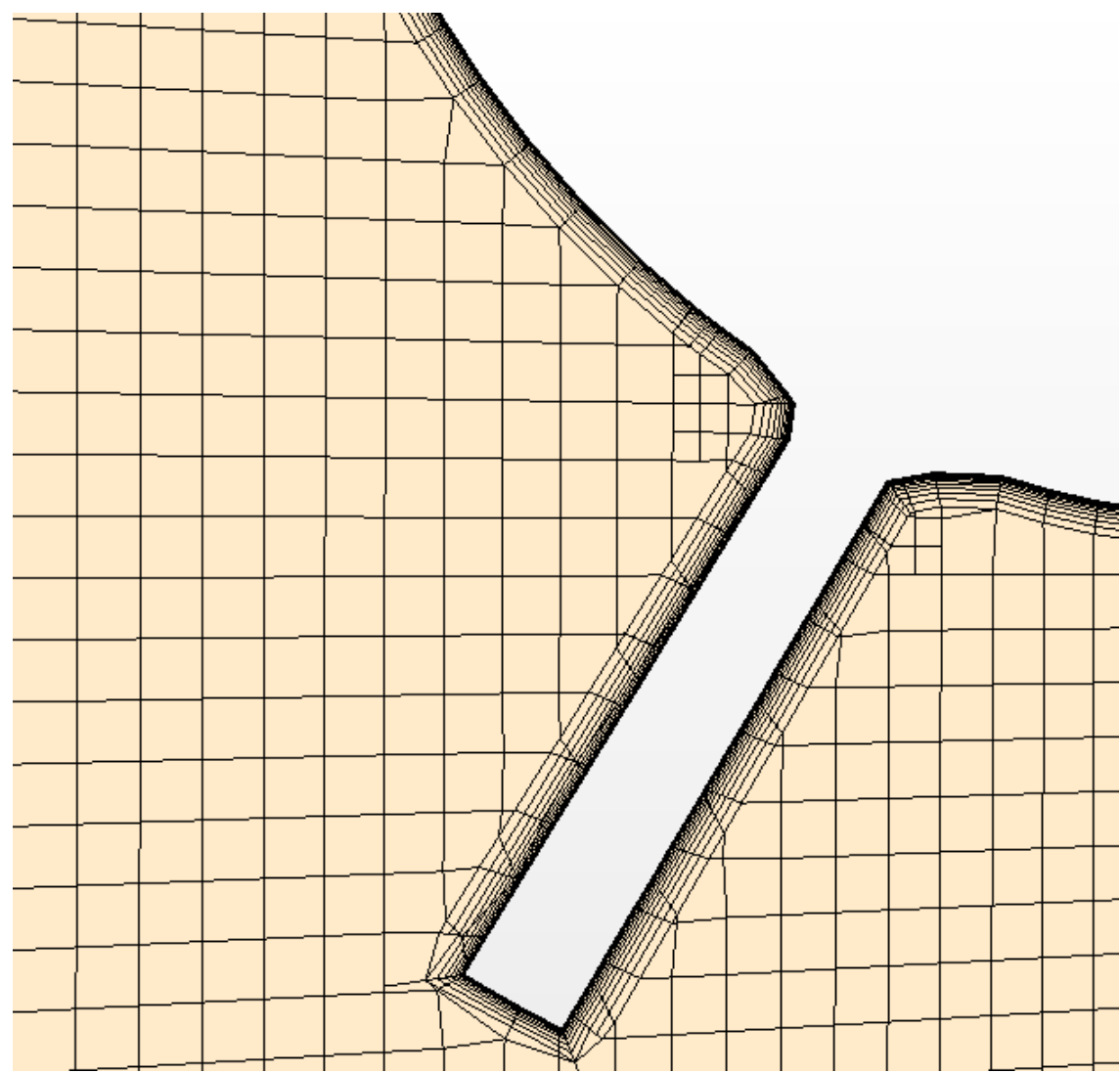

Figure $18 \mathrm{~A}$ view of the prism layer generation around column and helical strakes 
This increase in boundary layer cells leads to an increase in the total cell count of this domain up to 6.8 million.

\subsection{Fourier analysis}

Fourier Series (FS) were used to post-process the unsteady time histories of the force coefficients under different current conditions. Each unsteady history $\varphi(t)$ can be represented by a Fourier Series in time as follows:

$$
\varphi(t)=\varphi_{0}+\sum_{n=1}^{N} \varphi_{n} \cdot \cos \left(2 \pi f n t+\gamma_{n}\right), \mathrm{n}=1,2,3, \ldots
$$

where $\varphi_{n}$ is the $n^{\text {th }}$ harmonic amplitude and $\gamma_{\mathrm{n}}$ is the corresponding phase. These values can be calculated using the following expressions:

$$
\begin{aligned}
& \varphi_{n}=\sqrt{a_{n}^{2}+b_{n}^{2}} \\
& \gamma_{n}=\arctan \left(\frac{b_{n}}{a_{n}}\right)
\end{aligned}
$$

in which,

$$
\begin{aligned}
& a_{n}=\frac{2}{T} \int_{0}^{T} \varphi(t) \cos (2 \pi f n t) d t \\
& b_{n}=-\frac{2}{T} \int_{0}^{T} \varphi(t) \sin (2 \pi f n t) d t
\end{aligned}
$$

In these equations $\mathrm{T}$ designates the simulation time. The $0^{\text {th }}$ harmonic amplitude $\varphi_{0}$ in FS is defined as the average value of the time history of $\varphi(t)$, which can be obtained as follows:

$$
\varphi_{0}=\frac{1}{T} \int_{0}^{T} \varphi(t) d t
$$

As explained by Tezdogan et al. (2015), the first FS harmonic $\varphi_{1}$ refers to the linear term from the unsteady histories. Hence, the zeroth and first FS harmonics have been named as the fundamental components in the linear system. In particular, taking precedence from previous studies, we focused on the $0^{\text {th }}$ and $l^{s t}$ order terms for the force coefficients in oscillation. During the post-processing of the quantities, it was observed that higher order terms have significant effects. This observation has also been reported in the literature. For example, Simonsen et al. (2013) claim that for resistance in waves, the second and third order FS terms may make up to 50 and $15 \%$ of the first order FS amplitude, 
respectively. For any further details regarding the higher order terms, reference can be made to Otzen and Simonsen (2010).

\section{Results}

The following section will outline the simulation results achieved during the research reported in this paper. This section is divided into three sub-sections, each of which focuses on different aspects of our results. Before proceeding to assess the obtained results, it is first necessary to undertake a proper verification study to assess the simulation numerical uncertainty. A similar verification study was also used and presented in Tezdogan et al. (2016).

\subsection{Verification study}

A verification study was undertaken to estimate the discretisation errors due to grid-size and time-step resolutions for a representative case (geometry without helical strakes, current speed: $1.5 \mathrm{~m} / \mathrm{s}$ ). It is expected that the numerical uncertainties for the other cases are of the same order.

Xing and Stern (2010) state that the Richardson extrapolation (RE) method (1911) is the basis for existing quantitative numerical error/uncertainty estimates for time-step convergence and grid-spacing. With this method, the error is expanded in a power series, with integer powers of grid-spacing or timestep taken as a finite sum. Commonly, only the first term of the series will be retained, assuming that the solutions lie in the asymptotic range. This practice generates a so-called grid-triplet study. Roache's (1998) grid convergence index (GCI) is useful for estimating uncertainties arising from gridspacing and time-step errors. Roache's GCI is recommended for use by both the American Society of Mechanical Engineers (ASME) (Celik et al., 2008) and the American Institute of Aeronautics and Astronautics (AIAA) (Cosner et al., 2006).

For estimating iterative errors, the procedure derived by Roy and Blottner (2001) was used. The results obtained from these calculations suggest that the iterative errors for both force coefficients are insignificant.

Grid-spacing and time-step convergence studies were carried out following the GCI method described in Celik et al. (2008). The convergence studies were performed with triple solutions using systematically refined grid-spacing or time-steps. For example, the grid convergence study was conducted using three calculations in which the grid size was systematically coarsened in all directions whilst keeping all other input parameters (such as time-step) constant. The mesh convergence analysis was carried out with the smallest time-step, whereas the time-step convergence analysis was carried out with the finest grid size.

To assess the convergence condition, the convergence ratio $\left(R_{k}\right)$ is used, as given by: 


$$
R_{k}=\frac{\varepsilon_{k 21}}{\varepsilon_{k 32}}
$$

where $\varepsilon_{k 21}=\varphi_{k 2}-\varphi_{k 1}$ and $\varepsilon_{k 32}=\varphi_{k 3}-\varphi_{k 2}$ are the differences between medium-fine and coarse-medium solutions, and $\varphi_{k 1}, \varphi_{k 2}$ and $\varphi_{k 3}$ correspond to the solutions with fine, medium and coarse input parameters, respectively. The subscript $\mathrm{k}$ refers to the $\mathrm{k}^{\text {th }}$ input parameter (i.e. grid-size or time-step) (Stern et al., 2006).

Four typical convergence conditions may be seen: (i) monotonic convergence $\left(0<R_{k}<1\right)$, (ii) oscillatory convergence $\left(R_{k}<0 ;\left|R_{k}\right|<1\right)$, (iii) monotonic divergence (Rk>1), and (iv) oscillatory divergence $\left(R_{k}<0 ;\left|R_{k}\right|>1\right)$. For diverging conditions (iii) and (iv), neither error nor uncertainty can be assessed (Stern et al., 2006). For convergence conditions, the generalized RE method is applied to predict the error and order-of-accuracy $\left(p_{k}\right)$ for the selected $k^{\text {th }}$ input parameter. For a constant refinement ratio $\left(r_{k}\right), p_{k}$ can be calculated by:

$p_{k}=\frac{\ln \left(\varepsilon_{k 32} / \varepsilon_{k 21}\right)}{\ln \left(r_{k}\right)}$

The extrapolated values can be calculated from Celik et al., (2008).

$\phi_{e x t}^{21}=\left(r_{k}^{p} \phi_{1}-\phi_{2}\right) /\left(r_{k}^{p}-1\right)$

The approximate relative error and extrapolated relative error can then be calculated using Equations 11 and 12, respectively (Celik et al., 2008):

$$
\begin{aligned}
& e_{a}^{21}=\left|\frac{\phi_{1}-\phi_{2}}{\phi_{1}}\right| \\
& e_{\text {ext }}^{21}=\left|\frac{\phi_{e x t}^{12}-\phi_{1}}{\phi_{e x t}^{12}}\right|
\end{aligned}
$$

Finally, the fine-grid convergence index is predicted by:

$$
G C I_{\text {fine }}^{21}=\frac{1.25 e_{a}^{21}}{r_{k}^{p}-1}
$$

It should be borne in mind that Equations 8-13 are valid for a constant $r_{k}$ value. Reference can be made to Celik et al. (2008) for the formulae valid for a non-constant refinement ratio. The notation style of this reference was used in this study in order to enable the verification results to be presented clearly.

For both the mesh-spacing and time-step convergence studies, a constant refinement ratio of $\sqrt{2}$ was chosen in this study. It is of importance to mention that during the mesh convergence study, the 
surface mesh properties on the semi-submersible geometry with the helical strakes appended were kept constant to model the structure accurately. Based on the mesh refinement ratio which was applied, the final mesh numbers for each mesh configuration are listed in Table 2. Similarly, the time-step convergence study was conducted with triple solutions using systematically lessened time-steps, starting from $(\Delta t=0.0042 L / U)$.

Table 2. The final cell numbers for each mesh configuration as a result of the mesh convergence study

\begin{tabular}{|l|l|}
\hline Mesh configuration & Total cell number \\
\hline Fine & $6,406,511$ \\
\hline Medium & $3,084,469$ \\
\hline Coarse & $1,541,630$ \\
\hline
\end{tabular}

The verification parameters of the force coefficients for the grid spacing and time-step convergence studies are presented in Tables 3 and 4, respectively.

Table 3. Grid convergence study for sinkage and total resistance coefficient

\begin{tabular}{|l|c|c|}
\hline & $\begin{array}{c}\text { Drag coefficient }\left(0^{\text {th }} \text { order FS harmonic) }\right. \\
\text { (with monotonic convergence) }\end{array}$ & $\begin{array}{c}\text { Sway force coefficient }\left(0^{\text {th }} \text { order FS harmonic) }\right. \\
\text { (with monotonic convergence) }\end{array}$ \\
\hline $\mathrm{r}$ & $\sqrt{2}$ & $\sqrt{2}$ \\
\hline$\varphi_{1}$ & 0.8064 & 0.0444 \\
\hline$\varphi_{2}$ & 0.7706 & 0.0411 \\
\hline$\varphi_{3}$ & 0.6025 & 0.0096 \\
\hline $\mathrm{R}$ & 0.213 & 0.105 \\
\hline $\mathrm{p}$ & 4.46 & 6.51 \\
\hline$\varphi_{\mathrm{ext}^{21}}$ & 0.81609 & 0.044786 \\
\hline $\mathrm{e}_{\mathrm{a}}{ }^{21}$ & $4.44 \%$ & $7.43 \%$ \\
\hline $\mathrm{e}_{\mathrm{ext}}{ }^{21}$ & $1.19 \%$ & $0.86 \%$ \\
\hline $\mathrm{GCI}_{\text {fine }}{ }^{21}$ & $1.50 \%$ & $1.09 \%$ \\
\hline
\end{tabular}

Table 4. Time-step convergence study for sinkage and total resistance coefficient

\begin{tabular}{|l|c|c|}
\hline & $\begin{array}{c}\text { Surge force coefficient } \\
\text { (with monotonic convergence) }\end{array}$ & $\begin{array}{c}\text { Sway force coefficient } \\
\text { (with monotonic convergence) }\end{array}$ \\
\hline $\mathrm{r}$ & $\sqrt{2}$ & $\sqrt{2}$ \\
\hline$\varphi_{1}$ & 0.8064 & 0.0444 \\
\hline$\varphi_{2}$ & 0.7658 & 0.0405 \\
\hline$\varphi_{3}$ & 0.6014 & 0.0085 \\
\hline $\mathrm{R}$ & 0.247 & 0.122 \\
\hline $\mathrm{p}$ & 4.04 & 6.07 \\
\hline$\varphi_{\mathrm{ext}}{ }^{21}$ & 0.81971 & 0.044947 \\
\hline $\mathrm{e}_{\mathrm{a}}^{21}$ & $5.04 \%$ & $8.78 \%$ \\
\hline $\mathrm{e}_{\mathrm{ext}^{21}} \mathrm{GCI}_{\mathrm{fine}}{ }^{21}$ & $1.62 \%$ & $1.20 \%$ \\
\hline
\end{tabular}


As can be seen from Tables 3 and 4, reasonably small levels of uncertainty were estimated for the obtained parameters. The numerical uncertainties in the finest-grid solution for drag and sway force coefficients are predicted as $1.50 \%$ and $1.09 \%$, respectively (Table 3). These values rise to $2.06 \%$ and $1.52 \%$, respectively, when calculating the numerical uncertainty in the smallest time-step solution (Table 4). It can be interpreted that the CFD modelling is more sensitive to the change in the time-step resolution.

\subsection{Drag and sway force coefficients}

Having performed the necessary verification study, the remainder of this section addresses the key findings of this study.

Table 5 presents a comparison of the $0^{\text {th }}$ and $1^{s t}$ order FS harmonics for the drag and sway force coefficients between the semi-submersible geometry with and without strakes at the three current speeds.

Table 5. The $0^{\text {th }}$ and $1^{\text {st }}$ order FS terms for the drag $\left(C_{x}\right)$ and sway force $\left(C_{z}\right)$ coefficients obtained from CFD

\begin{tabular}{|c|c|c|}
\hline \multirow{2}{*}{ Current Condition } & \multicolumn{2}{|c|}{ Semi-submersible geometry } \\
\hline & without strakes & with strakes \\
\hline \multicolumn{3}{|c|}{ Current speed: $0.5 \mathrm{~m} / \mathrm{s}$ [Re: 10264878$]$} \\
\hline $0^{\text {th }}, 1^{\text {st }} \mathrm{C}_{\mathrm{x}}$ & $0.8976,0.1191$ & $1.1502,0.0024$ \\
\hline $0^{\text {th }}, 1^{\text {st }} \mathrm{C}_{\mathrm{z}}$ & $0.0116,0.0490$ & $-0.0028,0.0181$ \\
\hline \multicolumn{3}{|c|}{ Current speed: $1.0 \mathrm{~m} / \mathrm{s}$ [Re: 20529757$]$} \\
\hline $0^{\text {th }}, 1^{\text {st }} \mathrm{C}_{\mathrm{x}}$ & $0.7809,0.0158$ & $1.1068,0.0157$ \\
\hline $0^{\text {th }}, 1^{\text {st }} \mathrm{C}_{\mathrm{z}}$ & $0.0635,0.0912$ & $-0.0419,0.0772$ \\
\hline \multicolumn{3}{|c|}{ Current speed: $1.5 \mathrm{~m} / \mathrm{s}$ [Re: 30794635$]$} \\
\hline $0^{\text {th }}, 1^{\text {st }} C_{x}$ & $0.8064,0.0214$ & $1.1017,0.0110$ \\
\hline $0^{\text {th }}, 1^{\text {st }} \mathrm{C}_{\mathrm{z}}$ & $0.0444,0.0430$ & $-0.0427,0.0374$ \\
\hline
\end{tabular}

As can be seen from Table 5, as the helical strakes appended to the hull increase the drag of the semisubmersible model, they are capable of reducing the mean and first order transverse forces acting on the structure. Another interesting result to be drawn from the table is that the helical strakes work more efficiently at lower Reynolds numbers in reducing the mean transverse forces when compared to those measured for a bare geometry (without any strakes).

The different flow characteristics in the semi-submersible geometry with and without helical strakes also lead to variations in the nondimensional $\mathrm{y}^{+}$values around the model. Figure 19 demonstrates the $\mathrm{y}^{+}$values around the semi-submersible geometry without and with strakes attached for a current speed of $1 \mathrm{~m} / \mathrm{s}$. As can be understood from Figure 19, at the same current speed condition, the wall $\mathrm{y}^{+}$values on the geometry with strakes are higher than those on the structure without any strakes. This stems 
from the higher drag values in the model with the strakes appended. A comparison of the relative cell velocity values on the semi-submersible geometry with and without strakes at the same current speed is made in Figure 20 to demonstrate the higher velocity values on the structure with strakes.
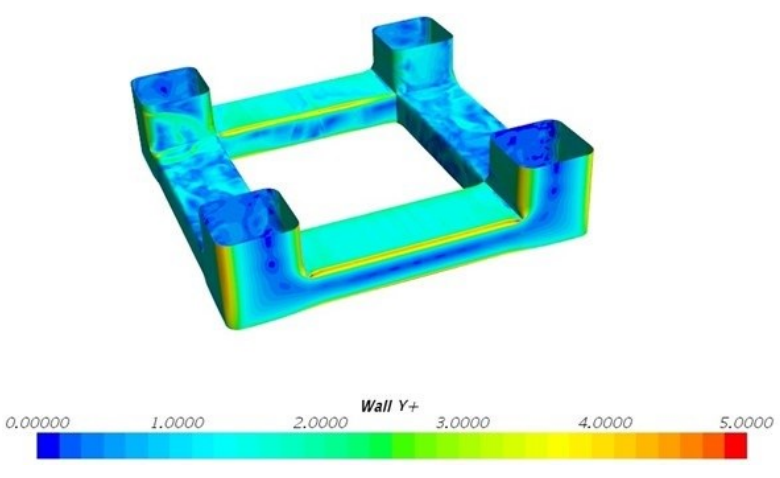
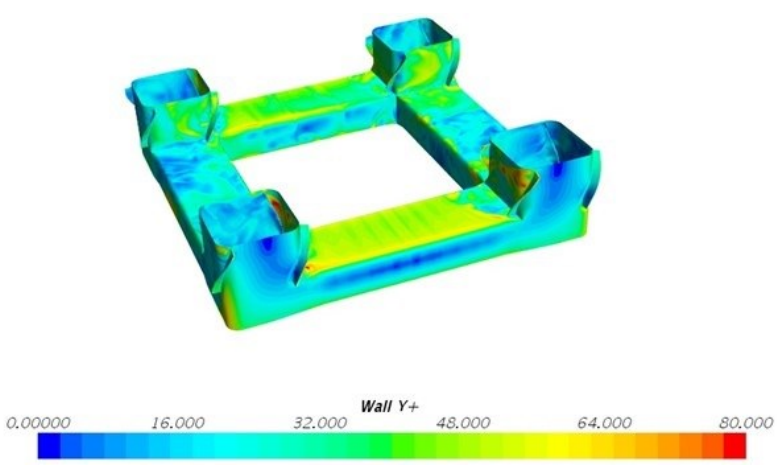

Figure 19. Wall $\mathrm{y}^{+}$distribution around the semi-submersible geometry without strakes (on the left) and with strakes (on the right) $\left(\right.$ Current speed $=1.0 \mathrm{~m} / \mathrm{s}, 0^{\circ}$ heading)
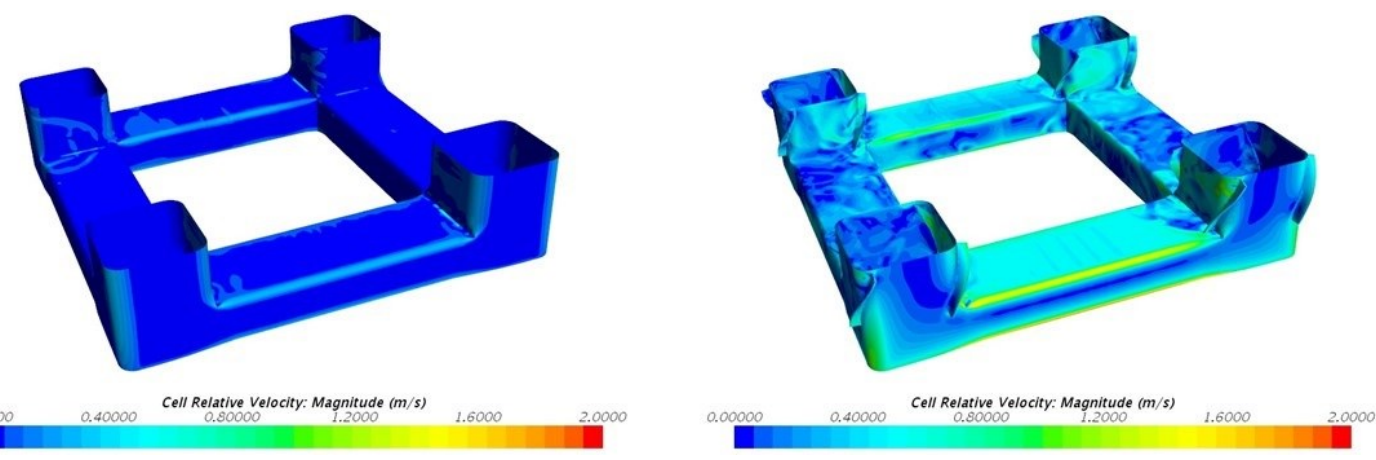

Figure 20. Cell relative velocity magnitudes around the semi-submersible geometry without strakes (on the left) and with strakes (on the right) (Current speed $=1.0 \mathrm{~m} / \mathrm{s}, 0^{\circ}$ heading)

\subsection{Vorticity}

In fluid mechanics, vorticity is defined as the tendency for fluid particles to spin. It is mathematically defined as the curl of the velocity vector. In fluid dynamics the distribution of vorticity is commonly used to characterise vortices, which are a major component of turbulent flow. This sub-section therefore aims to show the effect of helical strakes to reduce the vorticity over the semi-submersible geometry.

Table 6 displays the mean surface average vorticity values for the two different models for the three current speeds. As can be seen from the table, the vorticity on the semi-submersible geometry increases with the increasing current velocity or Reynolds number. This is as expected given that the higher the Reynolds number the stronger turbulent flow. That is to say, flows become more turbulent at higher Reynolds numbers. Table 6 also shows the mean vorticities for all of the current speeds are reasonably reduced when the helical strakes are appended to the semi-submersible hull. 
Table 6. The mean surface average vorticity values for the semi-submersible model at different current speeds

\begin{tabular}{|c|c|c|}
\hline \multirow{2}{*}{ Current velocity $(\mathbf{m} / \mathbf{s})$} & \multicolumn{2}{|c|}{ Mean surface average vorticity $(/ \mathbf{s})$} \\
\cline { 2 - 3 } & Without strakes & With strakes \\
\hline 0.5 & $3.05 \times 10^{2}$ & $7.87 \times 10^{1}$ \\
\hline 1 & $9.62 \times 10^{2}$ & $1.72 \times 10^{2}$ \\
\hline 1.5 & $1.93 \times 10^{3}$ & $2.62 \times 10^{2}$ \\
\hline
\end{tabular}

Figure 21 shows the vorticity distribution in magnitude over the semi-submersible model with and without strakes at a current speed of $1.5 \mathrm{~m} / \mathrm{s}$. As seen in the figure, the helical strakes work efficiently to reduce the vorticities that occur due to the turbulent behaviour of the fluid flow. Figure 22 demonstrates the vorticity contours for the semi-submersible with and without helical strakes at a current speed of $1.5 \mathrm{~m} / \mathrm{s}$. Without helical strakes, it can clearly be seen from the vorticity contours that relatively symmetrical vortex shedding occurs around the upstream columns, promoting VIM. On the other hand, if helical strakes are in place, then vortex shedding occurs at the edges of the strakes, resulting in less symmetrical vortex shedding from the upstream columns. The present findings are consistent with the results of Tan et al. (2014a). It should also be mentioned that Figure 21 and Figure 22 were obtained when the simulations had completed their run.
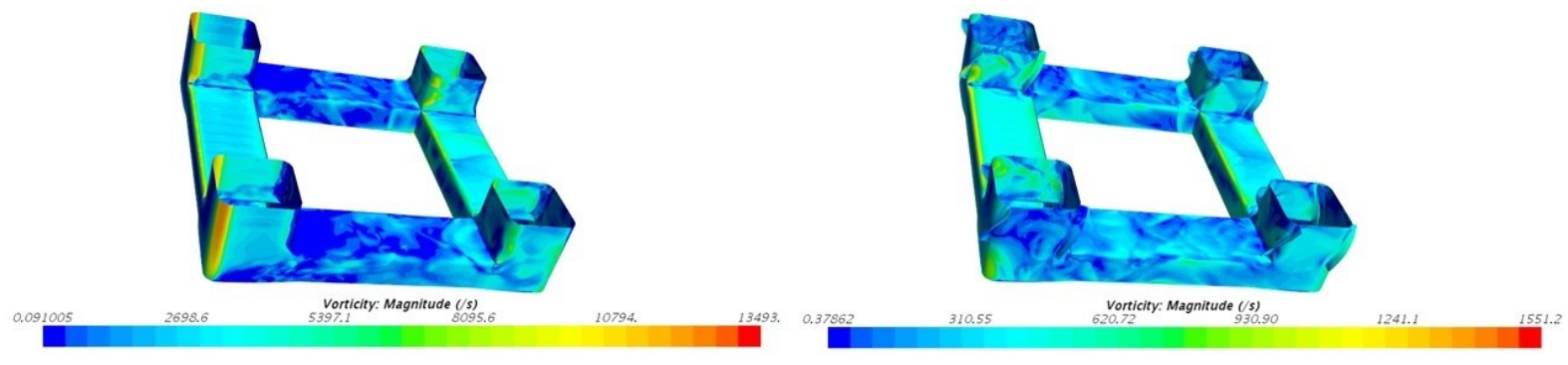

Figure 21. Vorticity magnitudes on the semi-submersible geometry without strakes (on the left) and with strakes (on the right) $\left(\right.$ Current speed $=1.5 \mathrm{~m} / \mathrm{s}, 0^{\circ}$ heading $)$
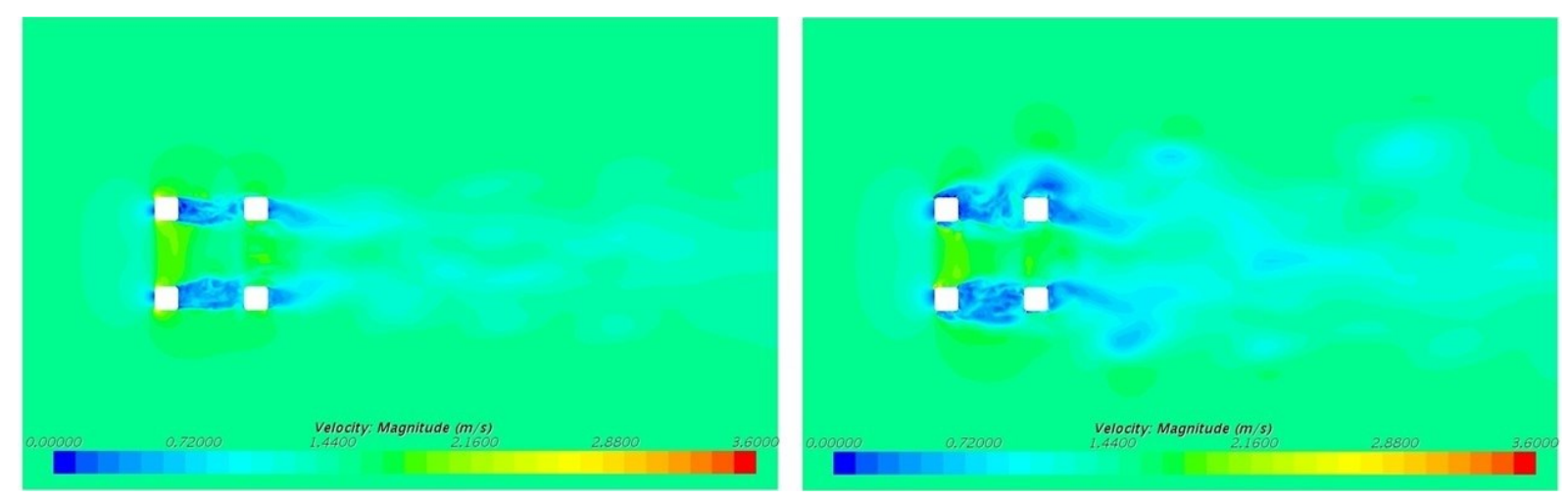

Figure 22. Vorticity contours for the semi-submersible model without strakes (on the left) and with strakes (on the right) $\left(\right.$ Current speed $=1.5 \mathrm{~m} / \mathrm{s}, 0^{\circ}$ heading) 


\section{Concluding remarks and future work}

In this paper, CFD calculations for a full-scale semi-submersible with and without helical strakes have been presented. The calculations have been carried out for three distinct current speeds that are prominent in the Gulf of Mexico due to its loop/eddy currents. To carry out the calculations, the finite volume code Star-CCM+ has been used with the DES model, which uses the RANS model inside the boundary region and LES model outside the boundary area. This allows a realistic simulation within the boundary region where the vortex shedding is taking place, while not using unnecessary amounts of computational power.

This paper started with a detailed literature survey in the area of VIMs focusing on state-of-the-art CFD-based techniques. It is expected that our literature review summarises the recent studies of researchers performing numerical and experimental studies in the area of VIM/VIVs. Later, the theoretical groundwork of this study was presented as complementary to the literature review. Following this, the numerical modelling of this work's CFD was explained in as much detail as possible. The figures related to the computational mesh from the domain were presented to complement our work's meshing strategy. After that, the formulations were given for the Fourier analysis to facilitate the post-processing of the CFD result. Later, in the results section, the verification study was conducted and the numerical uncertainty results were provided in a standard tabular format. The results suggest very small uncertainties, especially for the mesh dependence test. The main simulation results show that the helical strakes are efficient in reducing the transverse forces whereas they increase the total drag of the geometry. In addition, the CFD results explicitly showed that the attachment of the helical strakes tend to reduce the vorticity on the semi-submersible model.

This paper has provided a good starting point for the analysis of the hydrodynamics of helical strakes when appended to an offshore structure. It might be argued that the attached strakes for this semisubmersible might be over dimensioned as it generated additional drag to the semi-submersible in question. The design should therefore be further analysed and optimised to achieve a good balance between increased drag and reduced transverse forces. Other designs could possibly be appendages or changes in the geometry of the columns, for example by adding volume in the middle of each column, thereby breaking up the coherence of vortexes shedding along the length of the column. An example of this is the 'Heave and VIM Suppressed' (HVS) semi-submersible proposed by Kyoung et al. (2015), adding volume at the bottom of the column to break up the coherence of vortex shedding along the column.

Accounting for damping and restoring forces like mooring lines and riser systems to the numerical modelling, as well as letting the geometry move freely within the domain, will generate a more realistic simulation. This would allow the analysis of more realistic vortex induced motions like sway and surge movements when including the effect of the vessel's own natural frequencies in the sway 
and surge directions. It is also worth investigating the influence of different current headings which can cause lock-in, potentially resulting in large and extreme motion responses.

\section{Acknowledgements}

The results were obtained using the EPSRC funded ARCHIE-WeST High Performance Computer (www.archie-west.ac.uk). EPSRC Grant no. EP/K000586/1. The authors would like to thank Miss Holly Yu for her help with the final proofreading. 


\section{REFERENCES}

Armin, M. (2016). Integrating of two tandem cylinders undergoing VIV: mathematical modelling and experiments. PhD Thesis, Naval Architecture, Ocean and Marine Engineering Department, University of Strathclyde, Glasgow.

Armin, M., Srinil, N. (2013) Wake-induced transverse vibration of two interfering cylinders in tandem arrangement: Modelling and analysis. $32^{\text {th }}$ International Conference on Ocean, Offshore and Arctic Engineering, France.

Atluri, S., Halkyard, J., Sirnivas, S. (2006). CFD Simulation of Truss Spar Vortex-Induced Motion. $25^{\text {th }}$ International Conference on Offshore Mechanics and Arctic Engineering, Hamburg, Germany.

Blevins, R.D. (1990). Flow-Induced Vibraion. Van Nostrand Reinhold Company, New York.

CD-Adapco, (2016). Star-CCM+ Documentation Version 11.02.

Celik, I.B., Ghia, U., Roache, P.J., Freitas, C.J. (2008). Procedure for estimation and reporting of uncertainty due to discretization in CFD applications. Journal of Fluids EngineeringTransactions of the ASME 130(7):078001, DOI: 10.1115/1.2960953

Cosner, R.R., Oberkampf, W.L., Rumsey, C.L., Rahaim, C.P., Shih, TIP (2006). AIAA Committee on standards for computational fluid dynamics: Status and plans. 44th Aerospace Sciences Meeting and Exhibit, Reno, Nevada

Dirac Delta Consultants Ltd, u.d. DiracDelta - science and engineering encyclopedia. Available at: http://www.diracdelta.co.uk/science/source/v/o/vortex\%20shedding/source.html [Accessed on 02/08/2016].

Ferziger, J. H., Peric, M. (2002). Computational Methods for Fluid Dynamics (Third Edit.). Springer, Berlin, Germany.

Gonçalves, R. T. et al., (2012). Wave effects on vortex-induced motion (VIM) of a large-volume semisubmersible platform. Rio de Janeiro, Brazil, ASME 2012 31st International Conference on Ocean, Offshore and Arctic Engineering.

Halkyard, J., Sirnivas, S., Holmes, S., Constantinides, Y., Oakley, O. and Thiagarajan, K., (2005). Benchmarking of truss spar vortex induced motions derived from CFD with experiments, OMAE 2005-67252, Halkidiki, Greece. 
Hong, Y., Choi, Y., Lee, J. \& Kim, Y., (2008). Vortex-induced motion of a deep-draft semisubmersible in current and waves. Vancouver, Canada, Eighteenth International Offshore and Polar Engineering Conference.

Irani, M. \& Finn, L., (2004). Model Testing for Vortex Induced Motions of Spar Platforms. 23rd International Conference on Offshore Mechanics and Arctic Engineering, OMAE'04-51315, Vancouver, B.C., Canada.

Irani, M. \& Finn, L., (2005). Improved Strake Design for Vortex Induced Motions of Spar Platforms. s.l., ASME 2005 24th International Conference on Offshore Mechanics and Arctic Engineering.

Kim, J.-W., Magee, A., Guan, K.Y.H. (2011). CFD simulation of flow-induced motions of a multicolumn floating platform, Proceedings of the ASME 2011 30th International Conference on Offshore Mechanics and Arctic Engineering, Rotterdam, The Netherlands, Paper no. OMAE2011-49437.

Kim, S.J., Spernjak, D., Holmes, S., Vinayan, V. \& Antony, A., (2015). Vortex-induced motion of floating structures: CFD sensitivity considerations of turbulence model and mesh refinements, ASME 34th International Conference on Offshore Mechanics and Arctic Engineering, Newfoundland, Canada, Paper no. OMAE2015-42221.

Kumar, R.A., Sohn, C.H., Gowda, B.H.L., (2008). Passive control of vortex-induced vibrations: An overview. Recent Patents on Mechanical Engineering 2008, 1, 1-11.

Kwon, S.H., Cho, J.W., Park, J.S., Choi, H.S., (2002). The effects of drag reduction by ribbons attached to cylindrical pipes. Ocean Engineering 29,1945-1958.

Kyoung, J. et al., (2015). Investigation on the VIM Mitigation of the HVS Semisubmersible. St. John's, Canada, ASME 2015 34th International Conference on Ocean, Offshore and Arctic Engineering.

Lefevre, C., Constantinides, Y., Kim, J.W., Henneke, M., Gordon, R., Jang, H., Wu, G., (2013). Guidelines for CFD simulations of spar VIM, ASME 32nd International Conference on Offshore Mechanics and Arctic Engineering, Nantes, France, Paper no. OMAE2013-10333.

Lundgren, J. \& Berg, A., (1982). Wave-included motions on a four-column semisubmersible. Texas, U.S.A, Offshore Technology Conference.

Ma, W., Mu, G., Thompson, H., Prislin, I., Maroju, S., (2013). Vortex induced motions of a column stabilized floater. Proceedings of the D.O.T International Conference, Houston, Texas, U.S.A. 
Nishino, T., Roberts, G.T., Zhang, X. (2008) Unsteady RANS and detached-eddy simulations of flow around a circular cylinder in ground effect. Journal of Fluids and Structures (24): 18-33, doi: doi:10.1016/j.jfluidstructs.2007.06.002.

Oey, L.-Y., Ezer, T. \& Lee, H.-C., (2002). Loop Current, Rings and Related Circulation in the Gulf of Mexico: A Review of Numerical Models and Future Challenges. I: Loop Current, Rings and Related Circulation in the Gulf of Mexico. s.1.:American Geophysical Union, pp. 31-50.

Otzen, J.F., Simonsen, C.D. (2010). Uncertainty assessment for KCS resistance and propulsion tests in waves. FORCE Technology, Report no. ONRIII187 01, Lyngby.

Richardson, L.F. (1911). The approximate arithmetical solution by finite differences of physical problems involving differential equations, with an application to the stresses in a Masonry dam. Philosophical Transactions of the Royal Society of London Series A-Containing Papers of a Mathematical or Physical Character 210:307-357, DOI: 10.1098/rsta.1911.0009

Rijken, O. \& Leverette, S., (2008). Experimental Study Into Vortex Induced Motion Response of Semi Submersibles With Square Columns. s.1., ASME 27th International Conference on Offshore Mechanics and Arctic Engineering.

Roache, P.J. (1998). Verification and Validation in Computational Science and Engineering. Hermosa Publishers, Albuquerque

Roddier, D., Finnigan, T. \& Liapis, S., (2009). Influence of the Reynolds Number on Spar Vortex Induced Motions (VIM): Multiple Scale Model Test Comparisons. Volume 5: Polar and Arctic Sciences and Technology; CFD and VIV, 1-5 June, pp. 797-806.

Roy, C.J., Blottner, F.G. (2001). Assessment of one- and two-equation turbulence models for hypersonic transitional flows. Journal of Spacecraft and Rockets 38(5):699-710

Sagaut, P., Deck, S. \& Terracol, M., (2013). Multiscale and Multiresolution Approaches in Turbulence: LES, DES and Hybrid RANS/LES Methods: Applications and Guidelines. Singapore: Imperial College Press.

Sengrupta, S. \& Chatterjee, M. K., (1986). Evaluation of Semisubmersible Motion Characteristics. Marine Technology, 3 July, pp. 217-225.

Simonsen, C.D., Otzen, J.F., Joncquez, S., Stern, F. (2013). EFD and CFD for KCS heaving and pitching in regular head waves. Journal of Marine Science and Technology, Vol. 18(4), pp. 435-459, doi: 10.1007/s00773-013-0219-0.

Spalart, P., Jou, W.-H., Strelets, M. \& Allmaras, S., (1997). Comments on the feasibility of LES for wings, and on hybrid RANS/LES approach. s.l., Advances in DNS/LES. 
Stern, F., Wilson, R., Shao, J., (2006). Quantitative V\&V of CFD simulations and certification of CFD codes. International Journal for Numerical Methods in Fluids 50(11): 1335-1355, DOI: 10.1002/fld.1090steaming.

Sumer, B. M. \& Fredsøe, J., (1997). Hydrodynamics Around Cylindrical Structures. s.1.:World Scientific Publishing Co. Pte. Ltd.

Tan, J.H.C.T., Magee, A., Kim, J.W., Teng, Y.J., Zukni, N.A. (2013). CFD simulation for vortex induced motions of a multi-column floating platform. Proceedings of the ASME 2013 32nd International Conference on Offshore Mechanics and Arctic Engineering, Nantes, France, Paper no. OMAE2013-11117.

Tan, J.H.C., Teng, Y.J., Magee, A., Ly, B.T.H. (2014a) The effect of appurtenances on the VIM performance of a TLP for Southeast Asia. Offshore Technology Conference Asia, Kuala Lumpur, Malaysia, Paper No. OTC-24871-MS.

Tan, J.H.C., Teng, Y.J., Magee, A., Ly, B.T.H., Aramanadka, S.B. (2014b) Vortex induced motion of TLP with consideration of appurtenances. Proceedings of the ASME 2014 33rd International Conference on Offshore Mechanics and Arctic Engineering, San Francisco, California, USA, Paper no. OMAE2014-23420.

Tezdogan T, Demirel YK, Kellett P, Khorasanchi M, Incecik A, Turan O (2015) Full-scale unsteady RANS CFD simulations of ship behaviour and performance in head seas due to slow steaming. Ocean Engineering, Vol. 36, pp. 186-206. DOI: 10.1016/j.oceaneng.2015.01.011

Tezdogan, T. (2015) Potential Flow and CFD-Based Hydrodynamic Analyses of Mono- and MultiHull Vessels. University of Strathclyde, Glasgow (Ph.D. thesis). http://ethos.bl.uk/OrderDetails.do?uin=uk.bl.ethos.647889

Tezdogan, T., Incecik, A., Turan, O. (2016). Full-scale unsteady RANS simulations of vertical ship motions in shallow water. Ocean Engineering, 123, 131-145.

DOI: 10.1016/j.oceaneng.2016.06.047.

Wang, Y., Yang, J., Peng, T. \& Li, X., (2009). Model test study on vortex-induced motions of a floating cylinder. s.1., ASME 2009 28th International Conference on Ocean, Offshore and Arctic Engineering.

White, F. M., (2006). Viscous Fluid Flow. Second Edition red. s.1.:McGraw-Hill, Inc.

Xing, T., Stern, F. (2010). Factors of Safety for Richardson Extrapolation. Journal of Fluids Engineering-Transactions of the ASME 132(6):061403, DOI: 10.1115/1.4001771. 
Xu, Q., Kim, J., Bhaumik, T., O’Sullivan, J., Ermon, J. (2012). Validation of HVS semisubmersible VIM performance by model test and CFD. Proceedings of the ASME 2012 31st International Conference on Offshore Mechanics and Arctic Engineering, Rio de Janeiro, Brazil, Paper no. OMAE2012-83207. 